\title{
Extremal Real Algebraic Geometry and $\mathcal{A}$-Discriminants ${ }^{1}$
}

\author{
Alicia Dickenstein* $\quad$ J. Maurice Rojas ${ }^{\dagger} \quad$ Korben Rusek ${ }^{\ddagger} \quad$ Justin $_{\text {Shih }}^{\S}$
}

December 15, 2006

For Askold Georgevich, with admiration and respect. Happy 60!

\begin{abstract}
We present a new, far simpler family of counter-examples to Kushnirenko's Conjecture. Along the way, we illustrate a computer-assisted approach to finding sparse polynomial systems with maximally many real roots, thus shedding light on the nature of optimal upper bounds in real fewnomial theory. We use a powerful recent formula for the $\mathcal{A}$-discriminant, and give new bounds on the topology of certain $\mathcal{A}$-discriminant varieties. A consequence of the latter result is a new upper bound on the number of topological types of certain real algebraic sets defined by sparse polynomial equations.
\end{abstract}

\section{Introduction}

The algorithmic study of real solutions of systems of multivariate polynomial equations is central in science and engineering, as well as in mathematics. For instance, entire fields such as Computer Aided Geometric Design and Control Theory essentially revolve on basic but highly non-trivial questions involving certain structured polynomial systems (see, e.g., [GK03, RS98]). Furthermore, polynomial systems whose real roots lie outside the reach of current algorithmic techniques regularly occur in a myriad of industrial problems, and many of these problems involve sparse polynomial systems, i.e., equations with "few" monomial terms. Understanding the number of real solutions of sparse polynomial equations is thus fundamentally important in real algebraic geometry.

Here we shed light on the difficulty behind determining the maximal number of real roots of polynomial systems with a fixed number of exponent vectors. We give new, dramatically simpler counter-examples (in Theorem 1.1 below) to an earlier conjectural upper bound of Kushnirenko. A consequence of our investigation is a precise quantitative statement that (for many fixed choices of exponent vectors) sparse polynomial systems with maximally many real roots are very rare: they lie in extremely small chambers - determined by a suitable discriminant variety — in the space of coefficients (Theorem 1.2 below). Moreover, we prove an explicit upper bound on the number of such chambers (see the proof of Theorem 1.3 below). This in turn implies a new upper bound on the number of smooth topological types attainable in families of real algebraic sets defined by certain sparse polynomials (Theorem 1.3 and Corollary 1.4 below).

${ }^{*}$ Departamento de Matemática, FCEN, Universidad de Buenos Aires, Cuidad Universitaria-Pabellón I, 1428 Buenos Aires, Argentina, alidick@dm.uba.ar . Partially supported by UBACYT X042, CONICET PIP 5617, and ANPCYT PICT 20569, Argentina.

${ }^{\dagger}$ Department of Mathematics, Texas A\&M University TAMU 3368, College Station, Texas 77843-3368, USA, rojas@math.tamu.edu , www.math.tamu.edu/ ${ }^{\sim}$ rojas . Partially supported by NSF CAREER grant DMS-0349309, NSF REU grant DMS-0243822, NSF individual grant DMS-0211458, and Sandia National Laboratories.

${ }^{\ddagger}$ Texas A\&M University, Department of Mathematics, TAMU 3368, College Station, TX 77843-3368, korben@rusek.org . Partially supported by the NSF REU program through grant DMS-0243822.

${ }^{\S}$ UCLA Mathematics Department, Los Angeles, CA 90095-1555, justin.shih@gmail.com . Partially supported by the NSF REU program through grant DMS-0243822.

${ }^{1}$ AMS Subject Classification: Primary 14P25, Secondary 14M25, 34C08. Keywords: sparse polynomial, real root, discriminant, isotopy, maximal, explicit bound. 
The techniques of our paper actually extend to general $\mathcal{A}$-discriminants and counting topological types of real zero sets of general sparse polynomials. The latter results will appear in a forthcoming paper. However, the special cases covered here already yield new results on extremal real algebraic geometry, which we now review in detail.

\subsection{Background on Extremal Estimates}

In a book published in June 1637, René Descartes stated that any real univariate polynomial with exactly $m$ monomial terms has at most $m-1$ positive roots [SL54]. Unlike the behavior of complex roots, Descartes' bound on the number of real roots is completely independent of the degree of the polynomial. However, nearly four centuries later, we still lack a definitive analogue for systems of multivariate polynomials. Great progress was made by Khovanskii and Sevastyanov [Kho80, Kho91] around the late 1970's, culminating in an explicit upper bound for the number of non-degenerate positive roots of general sparse polynomial systems. This bound - a very special case of Khovanskii's Theorem on Real Fewnomials [Kho91] - revealed that the maximal number of isolated real roots of polynomial systems with a fixed number of exponent vectors is independent of the sizes of the exponent vectors. Khovanskii's theory has since enabled important advances in many different areas, e.g., Hilbert's 16 $\frac{\text { th }}{}$ Problem [Kal03], algorithmic complexity [GV01, VG03, BRS06], the study of torsion points on algebraic curves [CZ02], and model theory [Wil99], to name but a few (see also the conclusion and bibliography of [Kho91]).

Khovanskii's original bound is now known to be far from tight (see, e.g., [LRW03, BS06]). More to the point, finding general optimal bounds is a decades-old problem whose solution would have significant impact outside, as well as inside, real algebraic geometry. Unfortunately, finding optimal bounds even for two equations in two unknowns - with just three terms each - turned out to be difficult enough to take close to 20 years to do.

To clarify this difficulty, consider the following polynomial system, which we will call the Haas system with parameters $(a, b, d)$ :

$$
H_{(a, b, d)}:=\left\{\begin{array}{l}
h_{1}(x, y):=x^{2 d}+a y^{d}-y \\
h_{2}(x, y):=y^{2 d}+b x^{d}-x
\end{array}\right.
$$

Letting $\mathbb{R}^{*}:=\mathbb{R} \backslash\{0\}$ and letting $\mathbb{R}_{+}$denote the positive ray, we call the collection of systems $\left\{H_{(a, b, d)}\right\}_{(a, b, d) \in\left(\mathbb{R}^{*}\right)^{2} \times \mathbb{N}}$ the Haas family.

Definition 1.1. Given any $f \in \mathbb{R}\left[x_{1}, \ldots, x_{n}\right]$ with $f(x)=\sum_{i=1}^{m} c_{i} x^{a_{i}}$ (where the $a_{i}$ are pair-wise distinct and all $c_{i}$ are nonzero) $)^{1}$ we call $f$ a (real) $n$-variate $m$-nomial. Also, given $f_{1}, \ldots, f_{k}$ with $f_{i}$ an $n$-variate $m_{i}$-nomial for all $i$, we call $F:=\left(f_{1}, \ldots, f_{k}\right)$ a $k \times n$ fewnomial system of type $\left(m_{1}, \ldots, m_{k}\right)$. Finally, if the total number of distinct exponent vectors among the $f_{i}$ is $m$, then we can also call $F$ an $m$-sparse $k \times n$ fewnomial system. $\diamond$

Thus, for example, any system from the Haas family can be referred to as (a) a $2 \times 2$ fewnomial system of type $(3,3)$, (b) a $2 \times 2$ trinomial system, or (c) a 6 -sparse $2 \times 2$ fewnomial system. Note also that $H_{(a, b, d)}$ has the same roots in $\left(\mathbb{R}^{*}\right)^{2}$ as $\left(h_{1}(x, y) / y, h_{2}(x, y) / x\right)$, which is 5 -sparse.

The aforementioned special case of Khovanskii's Theorem on Real Fewnomials (invoking an improvement observed by Daniel Perrucci [Per05]) states that an $m$-sparse $n \times n$ fewnomial system never has more than $(n+1)^{m-1} 2^{(m-1)(m-2) / 2}$ non-degenerate roots in the positive orthant $\mathbb{R}_{+}^{n}$. This in turn implies that the maximal number of non-degenerate roots in $\mathbb{R}_{+}^{2}$ of any $H_{(a, b, d)}$ in the Haas family is no more than 5184, since we can replace any $H_{(a, b, d)}$ by a 5 -sparse system with the same roots in $\mathbb{R}_{+}^{2}$ (cf. the preceding paragraph).

\footnotetext{
${ }^{1}$ For any $\alpha \in \mathbb{R}^{n}$, the notation $\alpha:=\left(\alpha_{1}, \ldots, \alpha_{n}\right)$ and $x^{\alpha}:=x_{1}^{\alpha_{1}} \cdots x_{n}^{\alpha_{n}}$ will be understood.
} 
Anatoly Kushnirenko, also around the late 1970s, conjectured a significant sharpening of Khovanskii's bound: Kushnirenko's Conjecture was the statement that $n \times n$ fewnomial systems of type $\left(m_{1}, \ldots, m_{n}\right)$ never have more than $\prod_{i=1}^{n}\left(m_{i}-1\right)$ non-degenerate roots in the positive orthant $\mathbb{R}_{+}^{n}$. This conjecture, if true, would have implied that the maximal number of non-degenerate roots in $\mathbb{R}_{+}^{2}$ of any $H_{(a, b, d)}$ in the Haas family is 4 , thus pointing to a rather large gap. (It is a simple exercise to construct $2 \times 2$ trinomial systems having 4 non-degenerate roots in $\mathbb{R}_{+}^{2}$.)

A bit of Gaussian elimination easily reveals that Kushnirenko's conjectural bound, if true, would have implied an elegant upper bound of $(m-n)^{n}$ for the number of non-degenerate roots of any $m$-sparse $n \times n$ fewnomial system [LRW03, Prop. 1]. Since $m$-sparse $n \times n$ fewnomial systems have no isolated roots in $\mathbb{R}_{+}^{n}$ when $m \leq n$ [LRW03, Prop. 1 and Thm. 4], the case where $n$ is fixed and $m$ is large becomes a natural question. Kushnirenko's Conjecture (or even an upper bound of the form $O(m)^{n^{2-\varepsilon}}$ for some $\left.\varepsilon>0\right)$ - if true - would have thus been a significant asymptotic improvement to Khovanskii's bound.

Remark 1.2. Curiously, over a different metrically complete field - the p-adic rationals $\mathbb{Q}_{p}$, for any fixed prime $p-i t$ is now known that the number of geometrically isolated roots in $\mathbb{Q}_{p}^{n}$ is $O((m-n) \log (m-n))^{3 n}$, for fixed $n$ and large $m$ [Roj04]. Also, in the complementary setting of fixed $m-n$ and large $n$, the number of non-degenerate roots in $\mathbb{R}_{+}^{n}$ is now known to be $O(n)^{m-n-1}[B S 06] . \diamond$

According to Bertrand Haas (and conversations of the second author with Dima Yu. Grigor'ev and Askold Khovanskii, on or before September 2000), Kushnirenko saw a simple counter-example to his conjecture shortly after he stated it in the late 1970's. Unfortunately, no one ever recorded this counter-example, or the identity of its author. Fortunately, Haas proved in 2000 [Haa02], via an ingenious elementary argument, that the system

$$
\begin{aligned}
& x^{106}+1.1 y^{53}-1.1 y \\
& y^{106}+1.1 x^{53}-1.1 x,
\end{aligned}
$$

along with many others with nearby real exponents, has at least 5 roots in $\mathbb{R}_{+}^{2}$. Shortly after, Li, Rojas, and Wang proved that all $2 \times 2$ trinomial systems (and, in particular, all systems in the Haas family) have at most 5 isolated roots in $\mathbb{R}_{+}^{2}$ [LRW03]. The latter trio of authors also significantly sharpened Khovanskii's bound for certain other families of $n \times n$ sparse polynomial systems.

Haas' example above was thus the simplest known counter-example to Kushnirenko's Conjecture, until the present paper.

Remark 1.3. It is interesting to observe that the existence of a pair of real bivariate polynomials $F:=\left(f_{1}, f_{2}\right)$ - with $f_{1}$ a trinomial and $f_{2}$ a tetranomial - having more than 6 isolated roots in $\mathbb{R}_{+}^{2}$, is still an open problem. The maximal number is known to be at least 6 , and no larger than 14 [LRW03, Thm. 1, Assertion (a)].

\subsection{Main Results}

\subsubsection{New Counter-Examples and the Probability of Finding One}

We give a new family of counter-examples far simpler than that of Haas, and announce what appear to be many more such families. In particular, while Haas found a pair of trinomials of degree 106 (and many more of higher degree), we given an explicit cell in $\mathbb{R}^{2}$ which is naturally identified with an infinite family of pairs of trinomials of degree $\mathbf{6}$. We have also found experimentally 49 other such cells (consisting of $2 \times 2$ systems of trinomial of even degree $<106$ ) which are all counter-examples to Kushnirenko's Conjecture as well, but we focus here on the simplest. 
Proposition 1.4. Let $E_{d} \subseteq \mathbb{R}^{2}$ denote the set of $(a, b)$ such that $H_{(a, b, d)}$ has at least 5 non-degenerate isolated roots in the positive quadrant. Then $E_{d}$ is open and symmetric about the line $\{a=b\}$.

Theorem 1.1. Following the notation of Proposition 1.4, $E_{3}$ is non-empty (and in fact star conve $\left.x^{2}\right)$. In particular, $\left(\frac{44}{31}, \frac{44}{31}\right) \in E_{3}$, and thus the $2 \times 2$ system $\left(x^{6}+\frac{44}{31} y^{3}-y, y^{6}+\frac{44}{31} x^{3}-x\right)$ has exactly 5 roots, all non-degenerate, in $\mathbb{R}_{+}^{2}$.

We point out that while our first verification of our simplest counter-example was done via Gröbner bases (on the computer algebra system Maple), we present here a novel (and simpler) numerical verification via Smale's Alpha Theory [Sma86, BCSS98].

Our next result reveals that for small $d$, it is highly unlikely that a random choice of $(a, b)$ will yield $H_{(a, b, d)}$ as a counter-example to Kushnirenko's Conjecture.

Theorem 1.2. Following the notation of Proposition 1.4, $E_{1}$ and $E_{2}$ are empty, and $0<\operatorname{Area}\left(E_{3}\right)<$ $5.701 \times 10^{-7}$. In particular, letting $a$ and $b$ be independent identically distributed standard real Gaussian random variables, $\operatorname{Prob}\left[(a, b) \in E_{3}\right]<1.936 \times 10^{-9}$. Finally, let $\alpha$ be the smallest positive root of the univariate degree 7 polynomial $C(a)$, which we define as

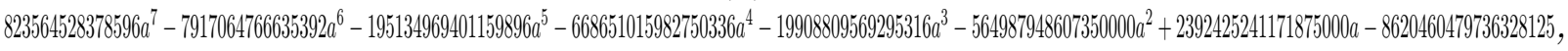
and let $\beta<\gamma$ be the two smallest positive roots of the univariate degree 36 polynomial $A(a)$ in the Appendix. Then the boundary of $E_{3}$ consists of exactly 4 convex arcs, meeting exactly at 4 vertices: To 10 decimal places, the preceding coordinates are

$$
\left\{\sqrt[5]{\alpha}\left[\begin{array}{l}
1 \\
1
\end{array}\right], \sqrt[5]{\frac{16807}{2916}}\left[\begin{array}{l}
1 \\
1
\end{array}\right],\left[\begin{array}{c}
\sqrt[35]{\beta} \\
\sqrt[35]{\gamma}
\end{array}\right],\left[\begin{array}{c}
\sqrt[35]{\gamma} \\
\sqrt[35]{\beta}
\end{array}\right]\right\}
$$

$$
\left\{1.4176759490\left[\begin{array}{l}
1 \\
1
\end{array}\right], 1.4195167977\left[\begin{array}{l}
1 \\
1
\end{array}\right],\left[\begin{array}{l}
1.4179051055 \\
1.4182147696
\end{array}\right],\left[\begin{array}{l}
1.4182147696 \\
1.4179051055
\end{array}\right]\right\} \text {. }
$$

This paucity of extremal examples can be visualized most easily by plotting the regions in the space of coefficients that yield $H_{(a, b, d)}$ with a given number of roots in $\mathbb{R}_{+}^{2} \cdot{ }^{3}$ Below is a sequence of 4 such plots (for $d=3$ ), drawn on a logarithmic scale and successively magnified up to a factor of about 1700 .
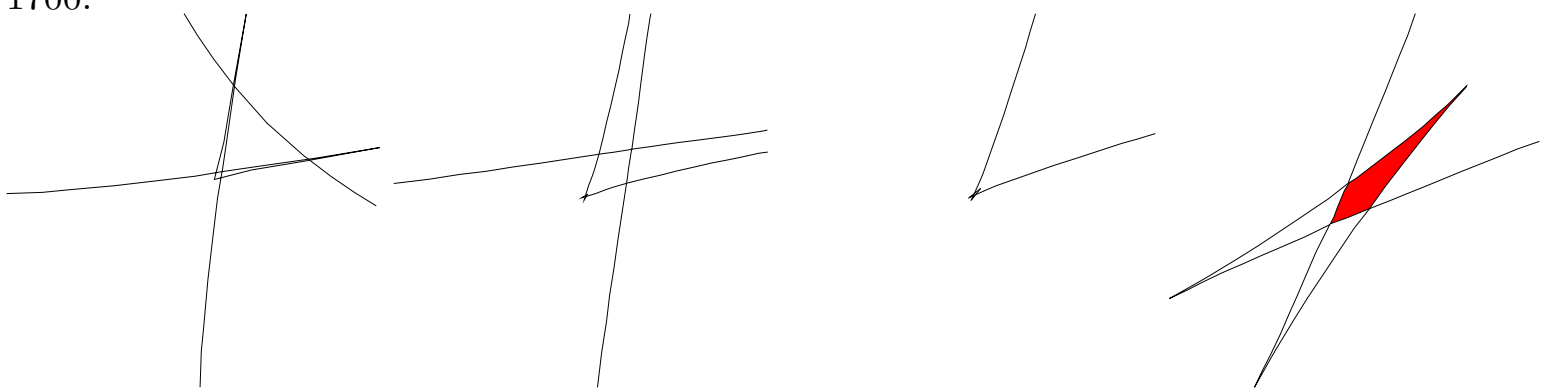

The small red (or grey) diamond-like region on the right-most plot is $E_{3}$. The connected components of the complement of the underlying real curve are regions on which the number of roots of $H_{(a, b, 3)}$ in $\mathbb{R}_{+}^{2}$ — as a function of $(a, b)$ - is constant (see also the discussion after the proof of Theorem 1.2 in Section 5 below). In particular, the real curve we see above is the real part of an $\mathcal{A}$-discriminant variety - a tool (reviewed in Section 2 below) underlying our final main result.

\footnotetext{
${ }^{2}$ Recall that a set $S \subset \mathbb{R}^{n}$ is star convex iff there is a $p \in S$ such that for all $x \in S$, the (closed) line segment containing $p$ and $x$ is contained in $S$ as well.

${ }^{3}$ The fact that systems in the Haas family have no roots on the coordinate cross, other than the origin, guarantees constancy (if we vary the coefficients while avoiding singularities) of the number of roots in each quadrant.
} 


\subsubsection{New Counts for Topological Types and Discriminant Chambers}

Recall that while a smooth, real, degree $d$ projective plane curve has at most $1+\left(\begin{array}{c}d-1 \\ 2\end{array}\right)$ connected components [Har76], determining the possible nestings of these ovals - a piece of the first part of Hilbert's famous $16^{\text {th }}$ Problem [Kal03] - is quite complicated. In more general language, this is the determination of possible diffeotopy types of such curves.

Definition 1.5. Recall that a diffeotopy between two sets $X, Y \subseteq \mathbb{R}^{n}$ is a differentiable function $H:[0,1] \times \mathbb{R}^{n} \longrightarrow \mathbb{R}^{n}$ such that $H(t, \cdot)$ is a diffeomorphism for all $t \in[0,1], H(0, \cdot)$ is the identity on $X$, and $H(1, X)=Y$. Equivalently, we simply say that $X$ and $Y$ are diffeotopic. $\diamond$

Note that diffeotopy is a more refined equivalence than diffeomorphism, since diffeotopy implies an entire continuous family of "infinitesimal" diffeomorphisms that deform $X$ to $Y$ and back again. Returning to nestings of ovals of real degree $d$ projective plane curves, an asymptotic formula of $e^{d^{2}}$ is now known [OK00], and the exact number is currently known (as of late 2006) only for $d \leq 8$.

Via our techniques here, we can study diffeotopy types in a dramatically different setting.

Definition 1.6. Given any n-variate m-nomial $f$, its support (or spectrum) - written $\operatorname{Supp}(f)$ - is its set of exponent vectors. Also, given any $k \times n$ fewnomial system $F=\left(f_{1}, \ldots, f_{k}\right)$, let $\operatorname{Supp}(F):=\left(\operatorname{Supp}\left(f_{1}\right), \ldots, \operatorname{Supp}\left(f_{k}\right)\right)$. Finally, we let $Z_{+}(F)$ (resp. $\left.Z_{\mathbb{R}}^{*}(F)\right)$ denote the set of roots of $F$ in $\mathbb{R}_{+}^{n}\left(\right.$ resp. $\left.\left(\mathbb{R}^{*}\right)^{n}\right)$.

Given $\mathcal{A} \subset \mathbb{Z}^{n}$ with $\# \mathcal{A}=n+3$, we let $Q$ denote its convex hull, and make a mild assumption that will be removed in future work (see, e.g., [BRS06, Sec. 3.2]): To avoid certain technicalities involving topological changes at infinity, we assume that the configuration $\mathcal{A}$ is generic in the sense that the intersection of $\mathcal{A}$ with each facet ${ }^{4}$ of $Q$ consists of exactly $n$ points. In particular, it is a routine exercise in polynomial/linear algebra to show that when the points of $\mathcal{A}$ are chosen uniformly randomly from $[-s, s]^{n} \cap \mathbb{Z}^{n}$, the failure probability of our genericity hypothesis decays like $O\left(1 / s^{n}\right)$ as $s \longrightarrow+\infty$.

Theorem 1.3. For any fixed $\mathcal{A} \subset \mathbb{Z}^{n}$ with $\# \mathcal{A}=n+3$ and satisfying the genericity assumption above, there are no more than $\left(10+6 n+n^{2}\right)\left(16+8 n+n^{3}+\frac{e^{2}+3}{2} \cdot(n+2)^{2}(n+4)^{2}\right)$ diffeotopy types for any smooth $Z_{\mathbb{R}}^{*}(f)$ with $\operatorname{Supp}(f)=\mathcal{A}$. In particular, the preceding bound (a) is no larger than $\frac{26}{5}(n+4)^{6}$, and (b) is completely independent of the coordinates of $\mathcal{A}$.

In contrast to the situation for degree $d$ plane curves, it is interesting to note that in our $n$-variate $(n+3)$-nomial setting, the number of possible diffeotopy types is much closer to the maximal number of compact connected components of $Z_{+}(f)$ : The latter number was recently shown to be no greater than $\left\lfloor\frac{5 n+1}{2}\right\rfloor[\mathrm{BS} 06]$. In view of Theorems 1.2 and 1.3, it thus appears that the intricacies of distinguishing attainable topological types for $Z_{+}(f)$ - for an $f$ with support $\mathcal{A} \subset \mathbb{Z}^{n}$ - might begin at $\# \mathcal{A}=n+3$.

In particular, for $\# \mathcal{A}=n+1$ (resp. $\# \mathcal{A}=n+2$ ), there are at most 2 (resp. 3) smooth diffeotopy types for $Z_{+}(f)$. The latter topological bounds are respectively proved in Section 3.2 and Theorem 2 of [BRS06], where the resulting algorithmic implications are pursued in much greater depth. We are unaware of any other earlier published explicit bounds - depending solely on $n$ and $\# \mathcal{A}-$ for the number of topological types of real fewnomial zero sets. That there are any such bounds at all is already a non-trivial fact, first observed by Lou van den Dries around the 1990s via o-minimality (see, e.g., [vdD98, Prop. 3.2, Pg. 150]). Thierry Zell has informed the authors that

\footnotetext{
${ }^{4}$ i.e., face of codimension 1
} 
results in [GVZ04] (on Pfaffian functions and quantifier elimination for fewnomials) appear to imply an upper bound of $n^{O\left(n^{2}\right)} 2^{O\left(n^{4}\right)}$ for the number of corresponding smooth topological types in our fewnomial setting above. ${ }^{5}$

Our polynomial bound above is thus a great improvement. It is also likely that our bound above can be improved even further: the main example we explore in this paper has $n=3$ and just 15 representative chambers, even though the underlying discriminant has over 58 monomial terms.

As a consequence of our last theorem, we get a constant upper bound for the number of smooth topological types of families of real algebraic surfaces defined by "honest" hexanomials.

Corollary 1.4. Fix $\mathcal{A} \subset \mathbb{Z}^{3}$, satisfying the assumption preceding Theorem 1.3 and with cardinality 6. Consider the family of polynomials $g$ with support $\mathcal{A}$, which we will identify with $\left(\mathbb{R}^{*}\right)^{6}$. Then the number of smooth diffeotopy types of any such $Z_{\mathbb{R}}^{*}(g)$ is no more than 237920.

Theorems 1.3, 1.1, and 1.2 are respectively proved in Sections 3, 4, and 5. The main underlying tools are an important recent parametric formula for certain $\mathcal{A}$-discriminant varieties [DFS05, Prop. 4.1], and recent advances in quantitative estimates for sheared binomial systems (cf. Section 2) [LRW03, BS06].

\section{Background on $\mathcal{A}$-Discriminants}

The standard reference for $\mathcal{A}$-discriminants is [GKZ94]. For our purposes, we will modify a few notions, present motivating examples, and quote some more recent results as well. But first, let us recall the following notation (see [Loe91] or [GKZ94, Ch. 1, 9-11] for further background).

Definition 2.1. Given any $\mathcal{A}=\left\{a_{1}, \ldots, a_{m}\right\} \subset \mathbb{Z}^{n}$ of cardinality $m$, we let $X_{\mathcal{A}}$ - the (projective) toric variety associated to $\mathcal{A}-$ be the closure of the parametrized subvariety $\left\{\left[t^{a_{1}}: \cdots: t^{a_{m}}\right] \mid t=\left(t_{1}, \ldots, t_{n}\right) \in\left(\mathbb{C}^{*}\right)^{n}\right\}$ of $\mathbb{P}_{\mathbb{C}}^{m-1}$. Finally, we define $\nabla_{\mathcal{A}}$ - the $\mathcal{A}$-discriminant variety - to be the closure of the set of all $\left[c_{1}: \cdots: c_{m}\right] \in \mathbb{P}_{\mathbb{C}}^{m-1}$ such that the hyperplane $\left\{c_{1} z_{1}+\cdots+c_{m} z_{m}=0\right\}$ intersects a regular point of $X_{\mathcal{A}}$ with a tangency. $\diamond$

$\nabla_{\mathcal{A}}$ also happens to be the closure of those $\left[c_{1}: \cdots: c_{m}\right] \in \mathbb{P}_{\mathbb{C}}^{m-1}$ such that the complex zero set of $\sum_{i=1}^{m} c_{i} x^{a_{i}}$ possesses a singularity in $\left(\mathbb{C}^{*}\right)^{n}$ [GKZ94, Prop. 1.1].

Example 2.2. It is a simple exercise to verify that $\nabla_{\{0,1,2\}}$ is the set of all $[a: b: c] \in \mathbb{P}_{\mathbb{C}}^{2}$ such that the quadratic polynomial $f(x):=a+b x+c x^{2}$ has degree $<1$ or a double root in $\mathbb{C}$. In particular, $\nabla_{\{0,1,2\}}$ is the projectivized complex zero set of $b^{2}-4 a c$, and one can check that the affine real zero set of $b^{2}-4 a c$ in $\mathbb{R}^{3}$ is a double cone. Topologically, $\mathbb{P}_{\mathbb{R}}^{2} \cap \nabla_{\{0,1,2\}}$ is thus a circle. $\diamond$

In particular, $\nabla_{\mathcal{A}}$ is an irreducible algebraic variety defined over $\mathbb{Z}$ (see, e.g., [GKZ94, Prop. 1.3, Pg. 15]), and this motivates the following important definition.

Definition 2.3. When $\operatorname{codim} \nabla_{\mathcal{A}}=1$, we define (up to sign) the $\mathcal{A}$-discriminant, $\Delta_{\mathcal{A}} \in \mathbb{Z}\left[c_{1}, \ldots, c_{m}\right]$, to be the (irreducible) defining polynomial of $\nabla_{\mathcal{A}}$. Otherwise, we set $\Delta_{\mathcal{A}}:=1$. $\diamond$

Example 2.4. Continuing our last example, we can take $\Delta_{\{0,1,2\}}=b^{2}-4 a c$. In particular, observe that for real $(a, b, c), f$ has exactly 0,1 , or 2 roots according as $\Delta_{\{0,1,2\}}(a, b, c)$ is negative, zero, or positive. Note also that the classical quadratic formula tells us that the roots of $f$ are differentiable functions of the coefficients $(a, b, c)$, provided $[a: b: c] \notin \nabla_{\{0,1,2\}} \cdot \diamond$

\footnotetext{
${ }^{5}$ As this paper was being finalized, Saugata Basu informed the authors that a similar (exponential) bound is likely also provable via [BV06], but with the advantage that the support can be varied when $n$ and $\# \mathcal{A}$ are fixed.
} 
Example 2.5. For $\mathcal{A}:=\{(0,0),(1,0),(2,0),(0,1)\}$, it is easily checked that $\nabla_{\mathcal{A}}$ is exactly $\left\{[a: b: c: d] \mid b^{2}-4 a c=d=0\right\}$ and thus $\Delta_{\mathcal{A}}=1 . \diamond$

$\mathcal{A}$-discriminants are central in computational algebraic geometry, containing all known multivariate resultants as special cases (see, e.g., [GKZ94, Prop. 1.7, pg. 274]). More to the point, they are notoriously difficult to compute: (1) detecting just their vanishing is NP-hard already for $\mathcal{A} \subset \mathbb{Z}^{2}$ [Pla84, Koi97], and (2) $\mathcal{A}$-discriminants can have many monomial terms, already for $\mathcal{A} \subset \mathbb{Z}$ and $\# \mathcal{A}=4$ [RY05, Sec. 1.2] (see also Example 2.9 of the next section).

Relevant to our applications, the fact that the vanishing of $\Delta_{\mathcal{A}}$ determines when certain hypersurfaces possess singularities readily implies that the real complement of $\nabla_{\mathcal{A}}$ can be used to encode the number of real roots of certain polynomial systems. In particular, let us call any connected component of $\mathbb{P}_{\mathbb{R}}^{\# \mathcal{A}-1} \backslash \nabla_{\mathcal{A}}$ a $(\mathrm{n})(\mathcal{A}$-)discriminant chamber. Lemma 2.22 of Section 2.2 below (see also [GKZ94, Ch. 11, Sec. 5]) relates the number of $\mathcal{A}$-discriminant chambers to the number of smooth topological types attainable by the real zero sets of certain families of sparse polynomial systems. Understanding discriminant chambers is thus a feasible route toward understanding the maximal number of real roots of $F$.

Remark 2.6. When $\# \mathcal{A} \in\{n+1, n+2\}$, $\mathcal{A}$-discriminant chambers turn out to have a very simple structure: just one (resp. at most two) chamber(s) for $\# \mathcal{A}=n+1$ (resp. $\# \mathcal{A}=n+2$ ) [GKZ94, Prop. 1.8, Pg. 274]. Hence our focus on $\# \mathcal{A}=n+3$ throughout this paper. $\diamond$

\subsection{Studying $\mathcal{A}$-Discriminant Chambers}

In what follows, we will always assume that $\mathcal{A}$ is a subset of $\mathbb{Z}^{n}$, of cardinality $m$, that affinely generates $\mathbb{Z}^{n}$. We will also frequently assume that $\mathbf{O} \in \mathcal{A}$; in which case $\mathcal{A}$ affinely generating $\mathbb{Z}^{n}$ is equivalent to $\mathbb{Z}^{n}$ being generated by the set of all integral linear combinations of the remaining vectors in $\mathcal{A}$. In general, these assumptions can easily be enforced by merely translating $\mathcal{A}$ and, if necessary, applying the Hermite factorization [Sto98] for integral matrices. $\mathcal{A}$-discriminants happen to be invariant (modulo a permutation of coordinates) under affine transformations of $\mathcal{A}$ that are injective and integral [GKZ94, Prop. 1.4, Pg. 272]. For now, let us observe a simple example.

Example 2.7. Note that while $\mathcal{A}:=\{3,30,57\}$ does not affinely generate $\mathbb{Z}$ or contain the origin, this $\mathcal{A}$ is a shifted multiple of a set that does both: $\{0,1,2\}$. In particular, the polynomial $a x^{57}+b x^{30}+c x^{3}$ has a degenerate root in $\mathbb{C}^{*}$ iff the polynomial $a x^{2}+b x+c$ has a degenerate root in $\mathbb{C}^{*}$. Thus, we clearly have $\nabla_{\{3,30,57\}}=\nabla_{\{0,1,2\}}$ and $\Delta_{\{3,30,57\}}=\Delta_{\{0,1,2\}}$.

Remark 2.8. We will frequently abuse notation by also identifying $\mathcal{A}$ with the $n \times m$ matrix $\left[\begin{array}{ccc}a_{11} & \cdots & a_{1 m} \\ \vdots & \ddots & \vdots \\ a_{n 1} & \cdots & a_{n m}\end{array}\right]$, where $a_{i}=\left[\begin{array}{c}a_{1 i} \\ \vdots \\ a_{n i}\end{array}\right]$ for all $i$. Also, given vectors $v_{1}, \ldots, v_{m} \in \mathbb{R}^{n}$, we will let $\left[v_{1}, \ldots, v_{m}\right]$ denote the $n \times m$ matrix whose $j^{\frac{\text { th }}{2}}$ column is $v_{j}$. Finally, we will use the notation $z^{\left[v_{1}, \ldots, v_{m}\right]}:=\left(z^{v_{1}}, \ldots, z^{v_{m}}\right) . \diamond$

The combinatorics of exponents will thus be particularly important throughout our development. Continuing this theme, we will define notions useful for simplifying discriminant chambers. However, before going into further definitions, let us first motivate the need for simplification via a more intricate $\mathcal{A}$-discriminant example.

Example 2.9. Suppose $\mathcal{A}:=\left\{\left[\begin{array}{l}6 \\ 0 \\ 0\end{array}\right],\left[\begin{array}{l}0 \\ 3 \\ 0\end{array}\right],\left[\begin{array}{l}0 \\ 1 \\ 0\end{array}\right],\left[\begin{array}{l}0 \\ 6 \\ 1\end{array}\right],\left[\begin{array}{l}3 \\ 0 \\ 1\end{array}\right],\left[\begin{array}{l}1 \\ 0 \\ 1\end{array}\right]\right\}$. The real part of $\nabla_{\mathcal{A}}$ is at the heart of Theorem 1.1, and it is interesting to observe that $\Delta_{\mathcal{A}}$ happens to be rather large: $\Delta_{\mathcal{A}}(1, a,-1,1, b,-1)$ is (up to sign)...

$1102507499354148695951786433413508348166942596435546875-516440160351044111358464119738658142157348733522052 a^{35}$ 
+ 54 additional monomial terms of comparable size

$-24519711093887016527058411574716512472434688 b^{46} a^{39}+82754024941868680778822139064668229594467072 b^{47} a^{33}$. In particular, $\Delta_{\mathcal{A}}\left(1, \frac{44}{31},-1,1, \frac{44}{31},-1\right) \neq 0$, and (via the proof of Lemma 2.22 below) this is equivalent to the fact that $H_{(44 / 31,44 / 31,3)}$ has no degenerate roots. In any event, it should be clear that we need a more efficient means of addressing $\nabla_{\mathcal{A}}$. $\diamond$

A beautiful recent (re)discovery is the fact that while $\Delta_{\mathcal{A}}$ can be unwieldy, $\nabla_{\mathcal{A}}$ always admits a compactly expressible parametrization: the Horn-Kapranov Uniformization (see also [Kap91, PT05]).

Theorem 2.10. [DFS05, Prop. 4.1] Given $\mathcal{A}:=\left\{a_{1}, \ldots, a_{m}\right\} \in \mathbb{Z}^{n}$, the discriminant locus $\nabla_{\mathcal{A}}$ is exactly the closure of

$$
\left\{\left[u_{1} t^{a_{1}}: \cdots: u_{m} t^{a_{m}}\right] \mid u:=\left(u_{1}, \ldots, u_{m}\right) \in \mathbb{C}^{m}, \mathcal{A} u=\mathbf{O}, \sum_{i=1}^{m} u_{i}=0, t=\left(t_{1}, \ldots, t_{n}\right) \in\left(\mathbb{C}^{*}\right)^{n}\right\} .
$$

Example 2.11. Continuing Example 2.9, it is easily checked that the set of vectors $\left\{\left(u_{1}, \ldots, u_{6}\right)\right\}$ needed to form the parametrization from Theorem 2.10 is a vector space with basis

$$
\{(-2,35,-33,-12,0,12),(-2,11,-9,-4,4,0)\} \text {. }
$$

We thus obtain that, in spite of the huge formula for $\Delta_{\mathcal{A}}(1, a,-1,1, b,-1)$ we saw earlier, $\nabla_{\mathcal{A}} \subset \mathbb{P}_{\mathbb{C}}^{5}$ is exactly the closure of

$$
\left\{\left[-(2 \lambda+2) t_{2}^{6}:(35 \lambda+11) t_{3}^{3}:-(33 \lambda+9) t_{3}:-(12 \lambda+4) t_{1} t_{3}^{6}: 4 t_{1} t_{2}^{3}: 12 \lambda t_{1} t_{2}\right] \mid \lambda \in \mathbb{C},\left(t_{1}, t_{2}, t_{3}\right) \in\left(\mathbb{C}^{*}\right)^{3}\right\} \cdot \diamond
$$

Computationally, however, we will need to express $\nabla_{\mathcal{A}}$ in an even more efficient manner. In particular, if we are studying the topology of the zero set of a polynomial as we vary its coefficients, we should certainly take advantage of the various homogeneities that preserve the topology of the underlying zero set.

Example 2.12. Returning to Examples 2.9 and 2.11, note that the set of exponent vectors of $g(x, y, v):=c_{1} x^{6}+c_{2} y^{3}+c_{3} y+v\left(c_{4} y^{6}+c_{5} x^{3}+c_{6} x\right)$ is exactly $\mathcal{A}$, and that the topology of $Z_{\mathbb{R}}^{*}(g)$ is preserved under nonzero scalings of the coefficient vector $\left(c_{1}, \ldots, c_{6}\right) \in\left(\mathbb{R}^{*}\right)^{6}$, and nonzero scalings of the variables $v, y, z$. In particular, if we would like to find $(\alpha, \beta, \gamma, \delta) \in\left(\mathbb{R}^{*}\right)^{4}$ such that $\delta g(\alpha x, \beta y, \gamma v)=x^{6}+a y^{3}-y+v\left(y^{6}+b x^{3}-x\right)$ for some real a and $b$, then we must clearly solve the binomial system

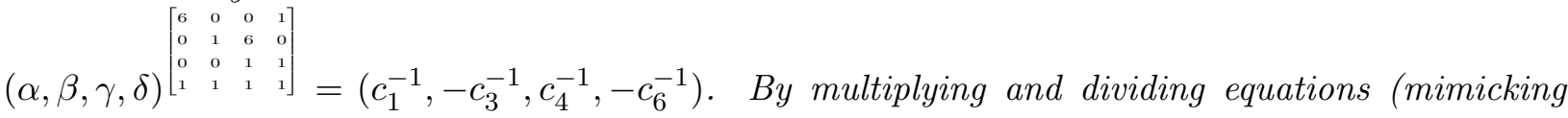
Gaussian elimination), one can then derive that

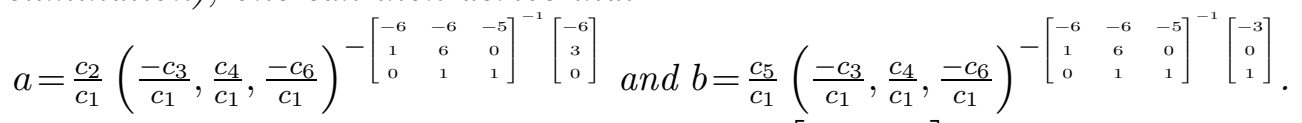

Note in particular that the determinants of the matrices $\left[\begin{array}{llll}6 & 0 & 0 & 1 \\ 0 & 1 & 6 & 0 \\ 0 & 0 & 1 & 1 \\ 1 & 1 & 1 & 1\end{array}\right]$ and $\left[\begin{array}{ccc}-6 & -6 & -5 \\ 1 & 6 & 0 \\ 0 & 1 & 1\end{array}\right]$ are both odd and differ only in sign. So one can check by hand (or via Lemma 2.13 and Proposition 2.17 below) that one can indeed always find such real $(a, b)$. More to the point, we have reduced the study of $Z_{\mathbb{R}}^{*}(g)$ from 6 to 2 parameters. $\diamond$

Lemma 2.13. Suppose $\mathcal{A}=\left\{a_{1}, \ldots, a_{m}\right\} \subset \mathbb{Z}^{n}$ affinely generates $\mathbb{Z}^{n}$ and $a_{1}=\mathbf{O}$. Then there are $i_{1}, \ldots, i_{n} \in\{2, \ldots, m\}$ such that $\operatorname{det}\left[a_{i_{1}}, \ldots, a_{i_{n}}\right]$ is odd.

Definition 2.14. Suppose $\mathcal{A}=\left\{a_{1}, \ldots, a_{m}\right\} \subset \mathbb{Z}^{n}$ affinely generates $\mathbb{Z}^{n}$, has cardinality $m \geq n+2$, and $a_{1}=\mathbf{O}$. We call any set $C=\left\{i_{1}, \ldots, i_{n}\right\}$ with $\operatorname{det}\left[a_{i_{1}}, \ldots, a_{i_{n}}\right]$ odd as in Lemma 2.13 above, an odd cell of $\mathcal{A}$. For any $n \times m$ matrix $B$, we then let $B_{C}$ (resp. $B_{C^{\prime}}$ ) denote the submatrix of $B$ 
defined by columns of $B$ with index in $C$ (resp. $\{2, \ldots, m\} \backslash C$ ). For any vectors $v, w \in\left(\mathbb{C}^{*}\right)^{m}$, let us denote their coordinate-wise product by $v \cdot w:=\left(v_{1} w_{1}, \ldots, v_{m} w_{m}\right)$. Also let $\Gamma$ be the multivalued 6 function from $\left(\mathbb{C}^{*}\right)^{m}$ to $\left(\mathbb{C}^{*}\right)^{m-n-1}$ defined by $\Gamma(y):=\frac{y_{C^{\prime}}}{y_{1}} \cdot\left(\frac{y_{C}}{y_{1}}\right)^{-A_{C}^{-1} A_{C^{\prime}}}$. Finally, we define the reduced $\mathcal{A}$-discriminant variety, $\bar{\nabla}_{\mathcal{A}} \subset \mathbb{C}^{m-n-1}$, to be the closure of

$$
\left\{\Gamma(u) \mid u:=\left(u_{1}, \ldots, u_{m}\right) \in\left(\mathbb{C}^{*}\right)^{m}, \mathcal{A} u=\mathbf{O}, \sum_{i=1}^{m} u_{i}=0\right\},
$$

and call any connected component of $\left(\mathbb{R}^{*}\right)^{m-n-1} \backslash \bar{\nabla}_{\mathcal{A}}$ a reduced $(\mathcal{A}$-)discriminant chamber. $\diamond$

Remark 2.15. Since we always implicitly assume that an odd cell has been fixed a priori for our reduced $\mathcal{A}$-discriminant varieties, $\Gamma$ in fact restricts to a single-valued function from $\left(\mathbb{R}^{*}\right)^{m}$ to $\left(\mathbb{R}^{*}\right)^{m-n-1}$.

Example 2.16. Continuing Examples 2.9, 2.11, and 2.12, let us shift our original $\mathcal{A}$ slightly to instead work with $\mathcal{A}=\left[\begin{array}{cccccc}0 & -6 & -6 & -6 & -3 & -5 \\ 0 & 3 & 1 & 6 & 0 & 0 \\ 0 & 0 & 0 & 1 & 1 & 1\end{array}\right]$. (The underlying $\mathcal{A}$-discriminants are left unchanged thanks to affine invariance [GKZ94, Prop. 1.4, Pg. 272].) As observed in Example 2.12, $C=\{3,4,6\}$ is an odd cell for $\mathcal{A}$. So $A_{C}$ is then the $3 \times 3$ matrix from Example 2.11, $A_{C^{\prime}}=$ $\left[\begin{array}{cc}-6 & -3 \\ 3 & 0 \\ 0 & 1\end{array}\right], A_{C}^{-1} A_{C^{\prime}}=\left[\begin{array}{cc}33 / 35 & -12 / 35 \\ 12 / 35 & 2 / 35 \\ -12 / 35 & 33 / 35\end{array}\right]$, and thus $\Gamma(u)=\left(\frac{u_{2} u_{6}^{12 / 35}}{u_{1}^{2 / 35} u_{3}^{33 / 35} u_{4}^{12 / 35}}, \frac{u_{5} u_{3}^{12 / 35}}{u_{1}^{12 / 35} u_{4}^{2 / 35} u_{6}^{33 / 35}}\right)$. Defining $\ell_{1}, \ldots, \ell_{6}$ respectively as the polynomials $-2 \lambda-2,35 \lambda+11,-33 \lambda-9,-12 \lambda-4,4,12 \lambda$, and letting $\Psi(\lambda):=\left(\psi_{1}(\lambda), \psi_{2}(\lambda)\right):=\Gamma\left(\ell_{1}(\lambda), \ldots, \ell_{6}(\lambda)\right)$, we thus obtain that $\bar{\nabla}_{\mathcal{A}}$ is the closure of $\left\{\Psi(\lambda) \mid \lambda \in \mathbb{C}, \ell_{1}(\lambda) \cdots \ell_{6}(\lambda) \neq 0\right\} . \diamond$

Let us now consider how the topology of $Z_{\mathbb{R}}^{*}(f)$ changes as $f$ ranges through $\mathcal{A}$-discriminant chambers. First, note that it is easy to show that for any $Z \subseteq\left(\mathbb{R}^{*}\right)^{n}$ and any coordinate reflection $\sigma: \mathbb{R}^{n} \longrightarrow \mathbb{R}^{n}, Z$ and $\sigma(Z)$ are not only diffeomorphic but diffeotopic. With just a little more work, one can then show the following:

Proposition 2.17. Suppose $\mathcal{A}=\left\{a_{1}, \ldots, a_{m}\right\} \subset \mathbb{Z}^{n}$ affinely generates $\mathbb{Z}^{n}$, has cardinality $m \geq n+2$, and $a_{1}=\mathbf{O}$. Also let $C$ be any odd cell of $\mathcal{A}$, let $f(x):=\sum_{i=1}^{m} \delta_{i} x^{a_{i}}$ with $\delta:=\left(\delta_{1}, \ldots, \delta_{m}\right) \in\left(\mathbb{R}^{*}\right)^{m}$, and let $\bar{\delta} \in\left(\mathbb{R}^{*}\right)^{m}$ be the unique vector with $\bar{\delta}_{1}=1, \bar{\delta}_{C}=(1, \ldots, 1)$ and $\bar{\delta}_{C^{\prime}}=\Gamma(\delta)$. Finally, let $\bar{f}:=\sum_{i=1}^{m} \bar{\delta}_{i} x^{a_{i}}$ and let $\operatorname{Conv} \mathcal{A}$ denote the convex hull of $\mathcal{A}$. Then:

1. $\Gamma$ induces a surjection from the set of connected components of

$$
\mathbb{P}_{\mathbb{R}}^{m-1} \backslash\left(\nabla_{\mathcal{A}} \cup\left\{\left[y_{1}: \cdots: y_{m}\right] \in \mathbb{P}_{\mathbb{R}}^{m-1} \mid y_{1} \cdots y_{m}=0\right\}\right)
$$

to the set of reduced $\mathcal{A}$-discriminant chambers.

2. If, for all facets $Q^{\prime}$ of $\operatorname{Conv} \mathcal{A}$, we have that $\#\left(\mathcal{A} \cap Q^{\prime}\right)=n$, then $Z_{\mathbb{R}}^{*}(f)$ and $Z_{\mathbb{R}}^{*}(\bar{f})$ are diffeotopic. Furthermore, for any $f_{1}$ and $f_{2}$ with $\bar{f}_{1}$ and $\bar{f}_{2}$ lying in the same reduced $\mathcal{A}$ discriminant chamber, $Z_{\mathbb{R}}^{*}\left(\bar{f}_{1}\right)$ and $Z_{\mathbb{R}}^{*}\left(\bar{f}_{2}\right)$ are diffeotopic.

Proposition 2.17 follows easily from a routine application of the Smith normal form and the implicit function theorem. In particular, the crucial trick is to observe that exponentiation by $A_{C}$, when $C$ is an odd cell, induces an automorphism of orthants of $\left(\mathbb{R}^{*}\right)^{n}$. Our assumption on the intersection of $\mathcal{A}$ with the facets of $\operatorname{Conv} \mathcal{A}$ ensures that any topological change in the zero sets of $f$ and $\bar{f}$ (in the underlying real toric variety corresponding to $\operatorname{Conv} \mathcal{A}\left[\right.$ Ful93]) occurs within $\left(\mathbb{R}^{*}\right)^{n}$.

Remark 2.18. It is also easily checked that our genericity assumption (on the intersection of $\mathcal{A}$ with the facets of $\operatorname{Conv} A$ ) implies that (a) the nullspace of $\mathcal{A}$ is not contained in any coordinate hyperplane, and (b) when $\# \mathcal{A}=n+3$, one has that $\bar{\nabla}_{\mathcal{A}} \cap \mathbb{R}^{2}$ is not contained in any line. $\diamond$

\footnotetext{
${ }^{6}$ The multiple values arise from the presence of rational exponents, and the number of images of a point is always bounded above by a constant depending only on $\mathcal{A}$.
} 
Definition 2.19. Following the notation of Definition 2.14, given any $\delta \in\left(\mathbb{C}^{*}\right)^{m}$, let $\delta^{\prime}$ be the unique vector with $\delta_{1}^{\prime}:=1, \delta_{C}^{\prime}=1$, and $\delta_{C^{\prime}}^{\prime}=\delta_{C^{\prime}}$. We then define the reduced $\mathcal{A}$-discriminant to be $\bar{\Delta}_{\mathcal{A}}\left(\delta_{C^{\prime}}\right):=\Delta_{\mathcal{A}}\left(\delta^{\prime}\right) . \diamond$

\subsection{Going Beyond One Polynomial Via the Cayley Trick}

We will need one last construction in order to apply $\mathcal{A}$-discriminants to systems of equations.

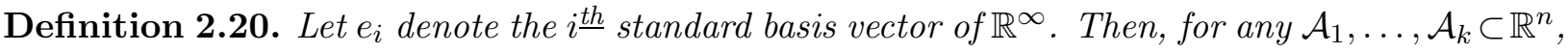
we call Cay $\left(\mathcal{A}_{1}, \ldots, \mathcal{A}_{k}\right):=\left(\mathcal{A}_{1} \times\{0\}\right) \cup\left(\mathcal{A}_{2} \times\left\{e_{n+1}\right\}\right) \cup \cdots \cup\left(\mathcal{A}_{k} \times\left\{e_{n+k-1}\right\}\right)$ the Cayley embedding of $\left(\mathcal{A}_{1}, \ldots, \mathcal{A}_{k}\right)$. We also define the Newton polytope of $f$ to be $\operatorname{Newt}(f):=\operatorname{Conv}(\operatorname{Supp}(f))$ and, for any compact set $B \subset \mathbb{R}^{n}$ and $w=\left(w_{1}, \ldots, w_{n}\right) \in \mathbb{R}^{n}$, we define $B^{w}$ - the face of $B$ with inner normal $w-$ to be $\left\{\left(x_{1}, \ldots, x_{n}\right) \in B \mid x_{1} w_{1}+\cdots+x_{n} w_{n}\right.$ is minimized $\} . \diamond$

Example 2.21. Taking $\left(\mathcal{A}_{1}, \mathcal{A}_{2}\right)$ to be the support of any $H_{(a, b, 3)}$ in the Haas family, observe that Cay $\left(\mathcal{A}_{1}, \mathcal{A}_{2}\right)$ is exactly the $\mathcal{A}$ from Examples 2.9-2.12. $\diamond$

Lemma 2.22. (See, e.g., [GKZ94, Prop. 1.7, Ch. 9 and Pg. 380].) Suppose $f_{1}, f_{2} \in \mathbb{R}[x, y]$ are bivariate polynomials with respective supports $\mathcal{A}_{1}$ and $\mathcal{A}_{2}$, and let $F:=\left(f_{1}, f_{2}\right)$. Assume also that for all $w \in \mathbb{R}^{2}$ the pair of faces $\left(\operatorname{Conv}\left(\mathcal{A}_{1}\right)^{w}, \operatorname{Conv}\left(\mathcal{A}_{2}\right)^{w}\right)$ never consists of two parallel edges. Then - identifying the coefficient of $x^{a}$ in $f_{i}$ with the point $(a, 0)$ or $(a, 1)$ of $\operatorname{Cay}\left(\mathcal{A}_{1}, \mathcal{A}_{2}\right)$ according as $i$ is 1 or 2 - the number of isolated real roots of $F:=\left(f_{1}, f_{2}\right)$ in any open quadrant is constant on any discriminant chamber of $\operatorname{Cay}\left(\mathcal{A}_{1}, \mathcal{A}_{2}\right)$.

Proof: Fixing an ordering on $\mathcal{A}_{1}$ and $\mathcal{A}_{2}$, let $c^{(i)}$ denote the coefficient vector of any $f_{i}$ with support $\mathcal{A}_{i}$. Also let $\nabla_{\left(\mathcal{A}_{1}, \mathcal{A}_{2}\right)}$ denote the closure in $\mathbb{P}_{\mathbb{C}}^{\# \mathcal{A}_{1}+\# \mathcal{A}_{2}-1}$ of those $\left[c^{(1)}: c^{(2)}\right]$ such that the corresponding $F:=\left(f_{1}, f_{2}\right)$ has a degenerate root in $\left(\mathbb{C}^{*}\right)^{2}$. Via [GKZ94, Prop. 1.7, Ch. 9 and Pg. $380], \nabla_{\left(\mathcal{A}_{1}, \mathcal{A}_{2}\right)}$ is exactly $\nabla_{\mathrm{Cay}\left(\mathcal{A}_{1}, \mathcal{A}_{2}\right)}$.

Note also that by our assumption on the $\left(\operatorname{Conv}\left(\mathcal{A}_{1}\right)^{w}, \operatorname{Conv}\left(\mathcal{A}_{2}\right)^{w}\right)$ (using $w \in\left\{e_{1}, e_{2}\right\}$ ), the only possible isolated root of $F$ on the coordinate cross is $(0,0)$. To conclude, we need only to observe that if $\left[c^{(i)}\right] \in \mathbb{P}_{\mathbb{C}}^{\# \mathcal{A}_{i}-1} \backslash \nabla_{\mathcal{A}}$ and $c^{(i)}$ has no zero coordinates for all $i \in\{1,2\}$, then all the roots of $F$ in the toric variety corresponding to $\operatorname{Conv}\left(\mathcal{A}_{1}\right)+\operatorname{Conv}\left(\mathcal{A}_{2}\right)$ lie in $\left(\mathbb{C}^{*}\right)^{2}$. Thus, along any fixed path within any fixed $\operatorname{Cay}\left(\mathcal{A}_{1}, \mathcal{A}_{2}\right)$-discriminant chamber, the roots of $F$ are continuous functions (with bounded range) of the coefficients.

\section{The Proof of Theorem 1.3}

The reduced $\mathcal{A}$-discriminant variety has many interesting properties that we will exploit. Before proving Theorem 1.3, however, we will need an important recent bound on the number of real roots of certain structured polynomial systems.

Definition 3.1. Suppose $\ell_{1}, \ldots, \ell_{j} \in \mathbb{R}\left[\lambda_{1}, \ldots, \lambda_{k}\right]$ are polynomials of degree $\leq 1$. We then call any system of equations of the form $S:=\left(1-\prod_{i=1}^{j} \ell_{i}^{b_{1, i}}\left(\lambda_{1}, \ldots, \lambda_{k}\right), \ldots, 1-\prod_{i=1}^{j} \ell_{i}^{b_{k, i}}\left(\lambda_{1}, \ldots, \lambda_{k}\right)\right)$, with $b_{i, i^{\prime}} \in \mathbb{R}$ for all $i, i^{\prime}$, and the vectors $\left(b_{1,1}, \ldots, b_{1, j}\right), \ldots,\left(b_{k, 1}, \ldots, b_{k, j}\right)$ linearly independent, $a$ $k \times k$ sheared binomial system with $j$ factors. We also call each $\ell_{i}$ a factor of the system. A sheared binomial system is referred to as a Gale Dual System in [BS06].॰

Note that our definition implies that $j \geq k$. For $j=k$, it is easy to reduce any $k \times k$ sheared binomial system with $j$ factors to a $k \times k$ linear system, simply by multiplying and dividing equations (mimicking Gaussian elimination). For $j>k$, sheared polynomial systems become much more complicated. 
Theorem 3.2. The number of non-degenerate roots $\lambda \in \mathbb{R}^{k}$ of any $k \times k$ sheared binomial system with $n+k$ factors, and all factors positive, is bounded above by:

1. [LRW03, Lemma 2] $n+1$ (and the same bound applies if we also count degenerate isolated roots with all factors positive), for $k=1$,

2. [BSO6] $\left(e^{2}+3\right) 2^{(k-4)(k+1) / 2} n^{k}$, for all $k \geq 1$.

In particular, $e^{2}+3 \approx 10.38905610$.

We will also need one last important result before our main proof. Let $\Psi(\lambda)=\Gamma\left(\ell_{1}(\lambda), \ldots, \ell_{m}(\lambda)\right)$ be the dense parametrization of a reduced $\mathcal{A}$-discriminant variety associated to an odd cell of $\mathcal{A}$ as in Definition 2.14. We can in fact consider this map defined over $\mathbb{P}_{\mathbb{C}}^{1}$ by considering the homogenizations $\ell_{i}\left(\left[\lambda_{1}: \lambda_{2}\right]\right):=\alpha_{i} \lambda_{1}+\beta_{i} \lambda_{2}$. Also, recalling Definition 2.19 and the fact that $\bar{\Delta}_{\mathcal{A}}$ is an irreducible defining polynomial for $\bar{\nabla}_{\mathcal{A}}$ with integer coefficients, let $Z$ be the finite set of points of $\bar{\nabla}_{\mathcal{A}}$ at which the gradient of $\bar{\Delta}_{\mathcal{A}}$ vanishes.

Lemma 3.3. With the above notation, if $\Psi\left(\left[\lambda_{1}, \lambda_{2}\right]\right) \in\left(\mathbb{R}^{*}\right)^{2} \cap\left(\bar{\nabla}_{\mathcal{A}} \backslash Z\right)$ then $\left[\lambda_{1}: \lambda_{2}\right]$ can be chosen in $\mathbb{P}_{\mathbb{R}}^{1}$. Then, except for a finite number of points, any point in $\mathbb{R}^{2} \cap \bar{\nabla}_{\mathcal{A}}$ is the image by $\Psi$ of a point with real coordinates.

Proof of Lemma 3.3: The proof is an easy consequence of the fact that $\Psi$ defines a multivalued function (univalued from the real points, as we have already noted in Remark 2.15) which is an inverse to the logarithmic Gauss map $G:\left(\mathbb{C}^{*}\right)^{2} \cap\left(\bar{\nabla}_{\mathcal{A}} \backslash Z\right) \longrightarrow \mathbb{P}_{\mathbb{C}}^{1}$ :

$$
G(y):=\left[y_{1} \frac{\partial}{\partial y_{1}} \bar{\Delta}_{\mathcal{A}}(y): y_{2} \frac{\partial}{\partial y_{2}} \bar{\Delta}_{\mathcal{A}}(y)\right]
$$

This is proved as in [CD06, PT04]. Now, since $G$ has rational coefficients, $G(y)$ has real coordinates for each real point $y \in\left(\mathbb{R}^{*}\right)^{2} \cap\left(\bar{\nabla}_{\mathcal{A}} \backslash Z\right)$.

We are now ready to prove Theorem 1.3 .

Proof of Theorem 1.3: Let $\mathcal{T}_{\mathcal{A}}$ denote the toric variety corresponding to the convex hull of $\mathcal{A}$ [Ful93]. Note that by our assumption that every facet of $\mathcal{A}$ corresponds to the vertices of a simplex, the complex zero set of any $f$ with $\operatorname{Supp}(f)=\mathcal{A}$ is thus always nonsingular at infinity, relative to $\mathcal{T}_{\mathcal{A}}$ (see, e.g., [BRS06, Sec. 3.2]). By Proposition 2.17, it then suffices to show that our desired bound applies to the number of reduced $\mathcal{A}$-discriminant chambers. Note also that by Proposition 2.17 and Lemma 3.3, the real part of the reduced $\mathcal{A}$-discriminant variety $-\mathbb{R}^{2} \cap \bar{\nabla}_{\mathcal{A}}$ - must be the union of a finite set of points and the closure of $\left\{\Psi(\lambda) \mid \lambda \in \mathbb{R}, \ell_{1}(\lambda) \cdots \ell_{n+3}(\lambda) \neq 0\right\}$, where $\Psi(\lambda):=\left(\psi_{1}(\lambda), \psi_{2}(\lambda)\right):=\left(\prod_{i=1}^{n+3} \ell_{i}^{b_{1, i}}(\lambda), \prod_{i=1}^{n+3} \ell_{i}^{b_{2, i}}(\lambda)\right)$, and $\ell_{1}, \ldots, \ell_{n+3}$ are univariate polynomials in $\lambda$ of degree $\leq 1$ Let $\Omega \subset \mathbb{R}^{2}$ denote the aforementioned closure. Since isolated points do not disconnect connected components of the complement of a (locally closed) real algebraic curve, it thus suffices to focus on $\Omega$. In particular, the connected components of $\left(\mathbb{R}^{*}\right)^{2} \backslash \Omega$ are (up to the deletion of finitely many points) exactly the reduced $\mathcal{A}$-discriminant chambers. Note also, by observing the poles of the $\psi_{i}$, that $\Omega$ is the closure of the union of no more than $n+4$ arcs, i.e., homeomorphic images of the open interval $(0,1)$.

To count the number of connected components of $\left(\mathbb{R}^{*}\right)^{2} \backslash \Omega$, we will use the classical critical points method [CG84], combined with our more recent tools. In particular, let us first bound the number of $x$-axis intersections, cusps, vertical tangents and vertical asymptotes, and nodes of $\Omega$ in $\mathbb{R}^{2}$. (These constitute all possible critical points of the orthogonal projection mapping $\Omega$ to the first coordinate axis.) Let us call the numbers of these respective objects $M_{0}(\mathcal{A}), M_{1}(\mathcal{A}), M_{2}(\mathcal{A})$, and $M_{3}(\mathcal{A})$, and proceed with bounding their number from above. 
$x$-axis Intersections: Clearly, $\Omega$ intersects the $x$-axis iff $\psi_{2}(\lambda)=0$, and the latter occurs iff a monomial in the $\ell_{i}$ vanishes at some $\lambda \in \mathbb{C} \cup\{ \pm \infty\}$. Also, by Theorem 2.10 and Definition 2.14, the degrees of the numerator and denominator of $\psi_{2}$ are equal. So there are clearly no more than $n+2$ solutions to $\psi_{2}(\lambda)=0$, and thus $M_{0}(\mathcal{A}) \leq n+2$.

Cusps (and certain isolated real points): To count cusps, it suffices to bound from above the number of complex $\lambda$ such that $\frac{\partial \psi_{1}}{\partial \lambda}=\frac{\partial \psi_{2}}{\partial \lambda}=0$. (Note also that for such a $\lambda, \Psi(\lambda)$ could also be an isolated real point of the real part of $\bar{\nabla}_{\mathcal{A}}$ off of $\Omega$.) In particular, via the product rule, and by dividing out by suitable monomials in $\ell_{1}(\lambda), \ldots, \ell_{n+3}(\lambda)$, the preceding equation reduces to a univariate system of the form $\sum_{i=1}^{n+3} \frac{b_{1, i} \ell_{i}^{\prime}(\lambda)}{\ell_{i}(\lambda)}=\sum_{i=1}^{n+3} \frac{b_{2, i} \ell_{i}^{\prime}(\lambda)}{\ell_{i}(\lambda)}=0$. We can then multiply through by $\ell_{1}(\lambda) \cdots \ell_{n+3}(\lambda)$ to obtain a univariate polynomial of degree $n+2$. Furthermore, it is easily checked that the maximal number of distinct cusps as $\lambda \longrightarrow \pm \infty$ is one, and thus $M_{1}(\mathcal{A}) \leq n+3$.

Vertical Tangents (and vertical asymptotes): Here, we proceed essentially the same as for cusps, but with only one derivative. However, $\lambda \in\{ \pm \infty\}$ can possibly yield two distinct vertical tangents. So $M_{2}(\mathcal{A}) \leq n+4$. Note also that this count includes all cusps.

Nodes: Here, we need to bound the number of 2-sets $\left\{\lambda, \lambda^{\prime}\right\}$ (so $\left.\lambda \neq \lambda^{\prime}\right)$ with $\Psi(\lambda)=\Psi\left(\lambda^{\prime}\right)$ and $\lambda, \lambda^{\prime} \in \mathbb{R} \cup\{-\infty,+\infty\}$. Counting these real pairs then reduces to counting the number of real solutions of a $2 \times 2$ sheared binomial system - with $\leq 2(n+3)=2 n+6$ factors - where no factor is zero. Theorem 3.2 counts such solution satisfying certain sign condition for the $\ell_{i}$, so let us carefully count the number of (nonzero) sign combinations possible for the vectors $\left(\ell_{1}(\lambda), \ldots, \ell_{n+3}(\lambda)\right)$ and $\left(\ell_{1}\left(\lambda^{\prime}\right), \ldots, \ell_{n+3}\left(\lambda^{\prime}\right)\right)$ : Clearly, each such a vector admits at most $(n+4)$ possible (nonzero) sign combinations, since the sign of any $\ell_{i}$ is constant to the right (or to the left) of its unique real root, and there are no more than $n+3$ real roots for our $\ell_{i}$. Thus, there are at most $(n+4)^{2}$ possibilities for the (nonzero) sign vector of $\left(\ell_{1}(\lambda), \ldots, \ell_{n+3}(\lambda), \ell_{1}(\lambda), \ldots, \ell_{n+3}(\lambda)\right)$. So, combining with Theorem 3.2 , and noting that there are $\leq(2 n+4)+2$ factors, we thus clearly obtain no more than $(n+4)^{2} \cdot\left(e^{2}+3\right) \cdot 2^{-2} \cdot(2 n+4)^{2}$ pairs $\left(\lambda, \lambda^{\prime}\right) \in \mathbb{R}^{2}$ with $\Psi(\lambda)=\Psi\left(\lambda^{\prime}\right)$ and $\lambda \neq \lambda^{\prime}$. Note also that there are infinitely many solutions of $\Psi(\lambda)=\Psi\left(\lambda^{\prime}\right)$ of the form $\lambda=\lambda^{\prime}$, but these are non-isolated and thus not counted by Theorem 3.2. So there are no more than $\frac{e^{2}+3}{2}(n+2)^{2}(n+4)^{2}$ nodes arising from 2 -sets in $\mathbb{R}^{2}$, since our underlying sheared system is symmetric.

Now, should a $\lambda \in \mathbb{R}$ yield $\Psi(\lambda)=\Psi( \pm \infty)$ as a node (for some fixed choice of sign), then $\psi_{2}\left(\lambda^{\prime}\right)$ must clearly have a well-defined nonzero limit as $\lambda^{\prime} \longrightarrow \pm \infty$, since we have already counted $x$-axis intersections and vertical tangents. We are thus reduced to counting the number of real roots of a univariate sheared binomial, with no factor zero. By Theorem 3.2 again (with $\leq(n+2)+1$ factors), and recalling our last observation on the sign vector of $\left(\ell_{1}, \ldots, \ell_{n+3}\right)$, we then directly obtain no more than $(n+4)(n+2)$ nodes arising from $\left(\lambda, \lambda^{\prime}\right) \in(\mathbb{R} \cup\{ \pm \infty\})^{2} \backslash \mathbb{R}^{2}$. (It is also easily checked that should $( \pm \infty, \mp \infty)$ yield a node, then there is another pair $\left(\lambda, \lambda^{\prime}\right) \in \mathbb{R}^{2}$ giving the same node.)

In summary, we thus obtain no more than $\frac{e^{2}+3}{2}(n+2)^{2}(n+4)^{2}+(n+2)(n+4)$ nodes, and thus $M_{3}(\mathcal{A}) \leq(n+2)(n+4)\left[1+\frac{e^{2}+3}{2} \cdot(n+2)(n+4)\right]$.

Back to Reduced Chambers... To count the number of connected components of $\left(\mathbb{R}^{*}\right)^{2} \backslash \Omega$, let us now introduce vertical lines $L_{1}, \ldots, L_{N}$ exactly at the locations of the $y$-axis, the $x$-axis intersections, the cusps, the vertical tangents, and the nodes of $\Omega$. Clearly, any connected component of

$$
T:=\left(\mathbb{R}^{*}\right)^{2} \backslash\left(\Omega \cup L_{1} \cup \cdots \cup L_{N}\right)
$$

is contained in a unique connected component of $\left(\mathbb{R}^{*}\right)^{2} \backslash \Omega$. So it suffices to count the connected components of $T$. To do the latter, observe that $N \leq 1+M_{0}(\mathcal{A})+M_{2}(\mathcal{A})+M_{3}(\mathcal{A})$ (since cusps were already counted among vertical tangents via our technique above), and that our lines $\left\{L_{i}\right\}$ thus di- 
vide $\left(\mathbb{R}^{*}\right)^{2}$ into no more than $1+N=1+1+(n+2)+(n+4)+(n+2)(n+4)\left[1+\frac{e^{2}+3}{2} \cdot(n+2)(n+4)\right]$ $=16+8 n+n^{2}+\frac{e^{2}+3}{2} \cdot(n+2)^{2}(n+4)^{2}$ vertical strips.

Now note that within the interior of each strip, $\Omega$ is smooth, does not intersect the $x$-axis, and has no vertical tangents. So to count components of $T$ within any particular vertical strip, we need only bound from above the number of non-degenerate intersections of $\Omega \cup\left\{x_{2}=0\right\}$ with a vertical line distinct from $L_{1}, \ldots, L_{N}$. This clearly reduces to counting the number of real roots of a binomial in $n+3$ univariate linear forms. Via our earlier sign condition count, and by Theorem 3.2 once again (with $\leq(n+2)+1$ factors), the desired upper bound is then $1+(n+4)(n+2)$. Thus, each of our vertical strips contains no more than $2+(n+2)(n+4)$ connected components of $T$. Taking into account the number of vertical strips, we thus finally arrive at an upper bound of

$$
\left(10+6 n+n^{2}\right)\left(16+8 n+n^{2}+\frac{e^{2}+3}{2} \cdot(n+2)^{2}(n+4)^{2}\right)
$$

for the number of connected components of $T$, our bound is proved.

To conclude, note that Assertion (b) follows immediately from our bound, and Assertion (a) follows from merely comparing coefficients in the underlying polynomials.

Remark 3.4. The diffeotopies we have used above are thus obtained by (i) following a path in a reduced $\mathcal{A}$-discriminant chamber, and (ii) performing a coordinate reflection. One could certainly allow a broader class of diffeotopies, and thus (potentially) greatly reduce the bound we have just proved. Diffeotopies obtained solely via (i) are analogues of what are sometimes called rigid diffeotopies in other settings (see, e.g., [OKO0]).

Remark 3.5. Isolated points can in fact occur in the real part of a reduced $\mathcal{A}$-discriminant variety. For instance, taking $\mathcal{A}=\left[\begin{array}{cccccc}0 & -6 & -6 & -6 & -3 & -5 \\ 0 & 3 & 1 & 6 & 0 & 0 \\ 0 & 0 & 0 & 1 & 1 & 1\end{array}\right]$ (as in Example 2.16), we will see in the next section (and the Appendix) that $\mathbb{R}^{2} \cap \bar{\nabla}_{\mathcal{A}}$ is the disjoint union of a connected finite union of smooth arcs and exactly 3 isolated points (located respectively in the,+--- , and -+ quadrants). This in turn implies that the real part of the (non-reduced) discriminant variety $\nabla_{\mathcal{A}}$ has connected components of codimension at least 2 , even though $\nabla_{\mathcal{A}}$ is itself codimension 1 and irreducible over $\mathbb{C}$. $\diamond$

\section{The Proof of Theorem 1.1}

Before going into our main proof, let us first review an important criterion for an "approximate" root to converge quickly under Newton iteration to a true root of a polynomial system.

Definition 4.1. [Sma86, BCSS98] Given any analytic function $F: \mathbb{C}^{n} \longrightarrow \mathbb{C}^{n}$, we let $F^{\prime}$ denote its Jacobian matrix, and define the Newton endomorphism, $N_{F}: \mathbb{C}^{n} \longrightarrow \mathbb{C}^{n}$ to be the function $N_{F}(z):=z-F^{\prime}(z)^{-1} F(z)$. Also, given any $z_{0} \in \mathbb{C}^{n}$, we define the sequence of Newton iterates of $z_{0}$ (under $F$ ) to be $\left(z_{n}\right)_{n \in \mathbb{N} \cup\{0\}}$ where $z_{n+1}:=N_{F}\left(z_{n}\right)$ for all $n \geq 0$. Finally, given any multi-linear operator $\mathcal{L}:\left(\mathbb{C}^{N}\right)^{k} \longrightarrow \mathbb{C}^{N}$ and $v=\left(v_{1} \ldots, v_{N}\right) \in \mathbb{C}^{N}$, we let $|v|:=\left|v_{1}\right|^{2}+\cdots+\left|v_{N}\right|^{2}$ denote the usual Hermitian norm and let $|\mathcal{L}|$ be the multi-linear operator norm $\max _{\left(v^{1}, \ldots, v^{k}\right) \in\left(\mathbb{C}^{N} \backslash \mathbf{O}\right)^{k}} \frac{\left|\mathcal{L}\left(v^{1}, \ldots, v^{k}\right)\right|}{\left|v^{1}\right| \ldots\left|v^{k}\right|} . \diamond$

Definition 4.2. [Sma86, BCSS98] Following the notation of Definition 4.1, we define the invariants $\beta(F, z):=\left|z-N_{F}(z)\right|=\left|F^{\prime}(z)^{-1} F(z)\right|, \gamma(F, z):=\sup _{k \geq 2}\left|\frac{1}{k !} F^{\prime}(z)^{-1} F^{(k)}(z)\right|^{1 /(k-1)}$, and $\alpha(F, z):=$ $\beta(F, z) \gamma(F, z)$. Also, let us call a point $z_{0} \in \mathbb{C}^{n}$ an approximate root of $F$ iff the Newton iterates of $z_{0}$ under $F$ satisfy $\left|\zeta-z_{n}\right| \leq\left(\frac{1}{2}\right)^{2^{n-1}}\left|\zeta-z_{0}\right|$ for all $n \geq 1$, for some true (and non-degenerate) root $\zeta \in \mathbb{C}^{n}$ of $F$. $\diamond$ 
Remark 4.3. Note that $F^{\prime}(z)^{-1} F^{(k)}$ is a symmetric $k$-linear operator from $\left(\mathbb{C}^{n}\right)^{k}$ to $\mathbb{C}^{n}$, and that the underlying coefficients can be identified with $k$ th order partial derivatives of the $f_{i}$ constituting $F$. In particular, observe that when $F$ is a polynomial system, the supremum in the definition of $\gamma\left(F, z_{0}\right)$ is in fact a maximum over a finite set with cardinality depending on the degrees of the $f_{i}$. $\diamond$

Note that approximate roots (as defined above) give an efficient, rigorous, and numerically feasible way to encode true roots: For instance, instead of specifying $n$ (likely huge) minimal polynomials for an algebraic point $\left(\zeta_{1}, \ldots, \zeta_{n}\right)$, and $n$ corresponding isolating intervals, we can instead simply give an $n$-tuple $\left(z_{0}^{1}, \ldots, z_{0}^{n}\right)$ that is an approximate root. One can then extract arbitrarily high accuracy through a small number of Newton iteration, thanks to Definition 4.2.

There has been much important work on proving useful complexity bounds for this approach (see, e.g., [CSMP03]). In the space here, we merely point to [BCSS98] as an excellent beginning reference. In particular, the $\alpha$ invariant gives a sufficient criterion to guarantee that a given point, and any point sufficiently near, is an approximate root.

Theorem 4.4. [BCSS98, Ch. 8] Following the notation above, suppose $z_{0} \in \mathbb{C}^{n}$ satisfies $\alpha\left(F, z_{0}\right)<$ 0.03. Then $z_{0}$ - and any point within distance $\frac{.05}{\gamma\left(F, z_{0}\right)}-i$ s an approximate root of $F$. Furthermore, the ball of radius $2 \beta\left(F, z_{0}\right)$ contains the unique root of $F$ to which the Newton iterates of $z_{0}$ converge.

The preceding result is sometimes referred to as a (robust) one point estimate, and considerably strengthens earlier seminal results of Kantorovich, from the 1960s, which relied on invariants defined over entire regions. We are now ready to prove Theorem 1.1.

Proof of Theorem 1.1: Let $\mathcal{H}(3)$ denote the Cayley embedding of the support of any $H_{(a, b, 3)}$ in the Haas family. Proposition 2.17 and Lemma 2.22 then tell us that we can find $E_{3}$ by using the critical points method [CG84], just as in the proof of Theorem 1.3. In particular, for the setting at hand, this reduces to the following four steps:

1. computing $R(a, b):=\bar{\Delta}_{\mathcal{H}(3)}(-a,-b)$, i.e., $\Delta_{\mathcal{H}(3)}(1, a,-1,1, b,-1)$ up to sign

2. computing the partial derivatives of $R$ up to order 2 (needed later to check vertical tangents and convexity of the underlying arcs)

3. isolating those real $a_{0}$ such that there is a real $b_{0}$ with $R\left(a_{0}, b_{0}\right)=\left.\frac{\partial R}{\partial b}\right|_{\left(a_{0}, b_{0}\right)}=0$

4. computing the number of roots of $H_{(a, b, 3)}$ in each quadrant, at representative choices of $(a, b)$, picking at least one representative pair from each reduced discriminant chamber

These computations are routine, albeit hours-long, via Maple. So we now summarize the crucial details.

Steps (1) and (2): We calculate $R$ by first computing the resultant $p$ (resp. $q$ ) of $h_{1}$ (resp. $h_{2}$ ) and the determinant of the Jacobian of $\left(h_{1}, h_{2}\right)$, with respect to the variable $y$, where $\left(h_{1}, h_{2}\right):=H_{(a, b, 3)}$. We then compute the resultant, $\widetilde{R} \in \mathbb{Z}[a, b]$, of $p$ and $q$ with respect to the variable $x$. $\widetilde{R}$ is a multiple of our desired $R$, and we can isolate $R$ by factoring $\widetilde{R}$ and picking out the factor of the correct degree. In particular, a quick volume calculation (see, e.g., [Roj03, Thm. 4.2.4]) shows that the degree of $R$ is bounded above by 236 , and there is only one such factor of $\widetilde{R}$.

It then turns out that $R$ is symmetric, with total degree 90 , degree 47 with respect to each variable, and exactly 58 monomial terms (each having a coefficient with between 43 and 56 decimal digits). In particular, up to sign, $R(a, b)$ is the polynomial from Example 2.9, and the full monomial term expansion of $R(a, b)$ can be downloaded from http://www . math. tamu. edu/ rojas/haas3disc 
Remark 4.5. It is also interesting to observe that $R(a, b)$ is of the form $r\left(a^{6} b^{-1}, b^{6} a^{-1}\right)$, where $r$ is a polynomial with total degree 18 and degree 9 with respect to each variable. $\diamond$

Step (3): Computing those real $a_{0}$ where both $R\left(a_{0}, b_{0}\right)$ and $\left.\frac{\partial R}{\partial b}\right|_{\left(a_{0}, b_{0}\right)}$ vanish can be done by computing the resultant of $\left(R, \frac{\partial R}{\partial b}\right)$, to eliminate the variable $b$. The resulting eliminant $X(a)$ is large, occupying a text file over $3 \mathrm{Mb}$ in size, but has just 4 non-monomial factors $-A^{*}, B^{*}$, $C^{*}$, and $D^{*}$, of respective degrees $1260,70,35$, and 5 - that have real roots. These factors are respectively of the form $A\left(a^{35}\right), B\left(a^{35}\right), C\left(a^{5}\right)$, and $D\left(a^{5}\right)$, for certain polynomials $A, B, C$, and $D$ of significantly lower degree: $D$ is exactly the minimal polynomial for the rational number $\frac{16807}{2916}, C$ is the degree 7 minimal polynomial for $\alpha$, and $A$ is the minimal polynomial for the real algebraic numbers $\beta$ and $\gamma$ (as mentioned in the statement of Theorem 1.2). $B(a)$ is the following quadratic polynomial:

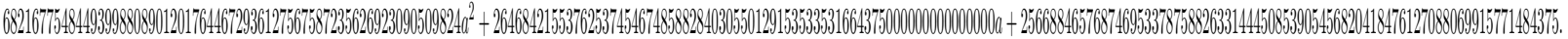

The critical values are thus the real roots of $A^{*}, B^{*}, C^{*}, D^{*}$, and there are exactly 16 of them: 2 of which are negative and 14 of which are positive. With a bit more work, it is then easily verified that the -+ and +- quadrants of $\mathbb{R}^{2}$ each contain exactly 2 reduced chambers (each unbounded), and the entire - - quadrant is itself a reduced chamber. Thus, all the action occurs in the ++ quadrant, and we will need to consider a total of $16+2+1$ vertical strips in our application of the critical points method to our real discriminant curve $\Omega:=\mathbb{R}^{2} \cap \bar{\nabla}_{\mathcal{H}(3)}$ (two extra vertical lines coming from the $b$-axis and the unique $a$-axis intersection of $\Omega$ ). The exact location of our vertical strips is detailed in the Appendix.

There turn out to be exactly 15 reduced chambers in $\mathbb{R}_{+}^{2}$ : exactly 5 that are unbounded and 10 that are bounded. An attractive illustration of the unbounded reduced chambers can be obtained by computing the logs of the absolute values of the coordinates of the zero set of $R$ in $\left(\mathbb{C}^{*}\right)^{2}$, i.e., the Archimedean amoeba of $R$ [GKZ94, Cor. 1.8]. In particular, the 5 unbounded reduced chambers (and the sole bounded chamber adjacent the origin, stretched to unboundedness - by the logarithm - in the illustration below), each contain exactly one of the white convex regions below. The remaining bounded chambers thus have images lying inside the shaded amoeba, and their boundaries are indicated by the darker curve: the image of $\mathbb{R}^{2} \cap \bar{\nabla}_{\mathcal{H}(3)}$ under the log of absolute value map.

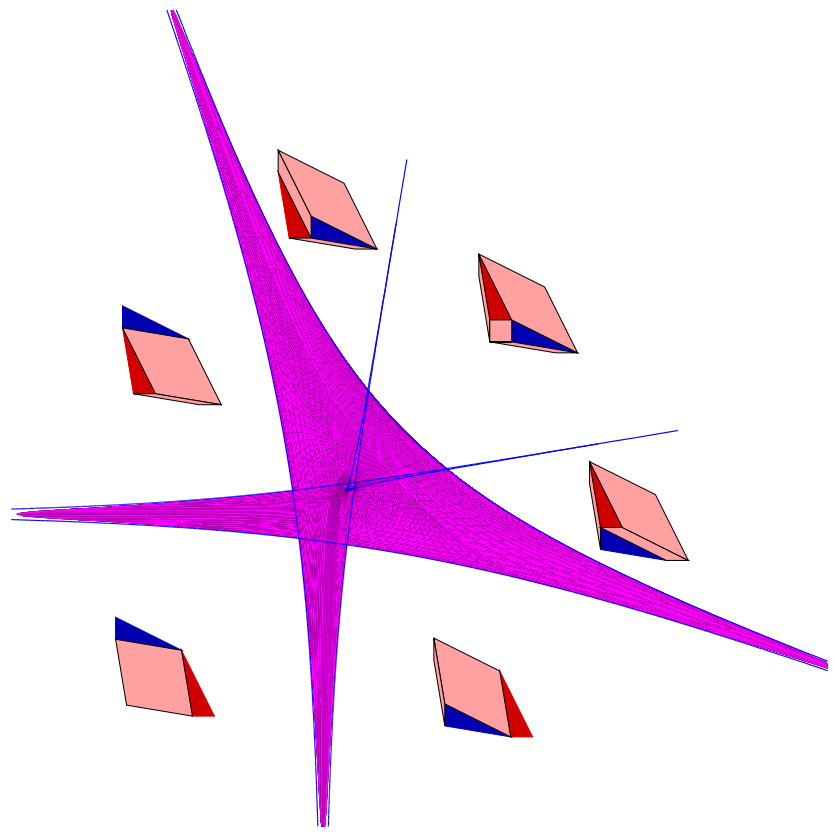


Remark 4.6. The Amoeba Theorem of Gelfand, Kapranov, and Zelevinsky [GKZ94, Cor. 1.8] tells us that the white convex regions above correspond exactly to the vertices of $\operatorname{Newt}(R)$. The fact that all the facets of the convex hull of $\mathcal{H}(3)$ are triangles implies that $\operatorname{Newt}(R)$ is actually the image of the secondary polytope of $\mathcal{H}(3)$ under an injective linear map [GKZ94, Thm. 1.7 of pg. 221 and Thm. 1.4 of pg. 302]. Concretely, this means that the white convex regions also correspond exactly to the triangulations of $\mathcal{H}(3)$, which we have drawn above as well. ${ }^{7} \diamond$

Step (4): Now let $T^{*}$ be the complement of $\Omega \cup L$ in $\left(\mathbb{R}^{*}\right)^{2}$, where $L$ is the set of vertical lines located at the real roots of the eliminant $X(a)$ computed in Step (3). Clearly then, each connected component of $T^{*}$ is contained in a unique reduced $\mathcal{H}(3)$-discriminant chamber.

Returning to the positive quadrant, exactly one of the reduced chambers there $\left(E_{3}\right)$ possesses $H_{(a, b, 3)}$ with 5 positive roots: all other chambers result in 4 or fewer positive roots. Verifying this reduces to solving a representative $H_{(a, b, 3)}$ for each connected component of $T^{*}$ : there are 125 such systems. In particular, after this sampling of representative points $(a, b)$, we obtain that $E_{3}$ is contained in the union of three adjacent vertical strips, with extreme end-points located approximately (to 10 decimal places) at $\{1.4176759490,1.4195167977\}$. We also obtain that within each strip, $E_{3}$ is the region between two smooth curves. This is illustrated below.

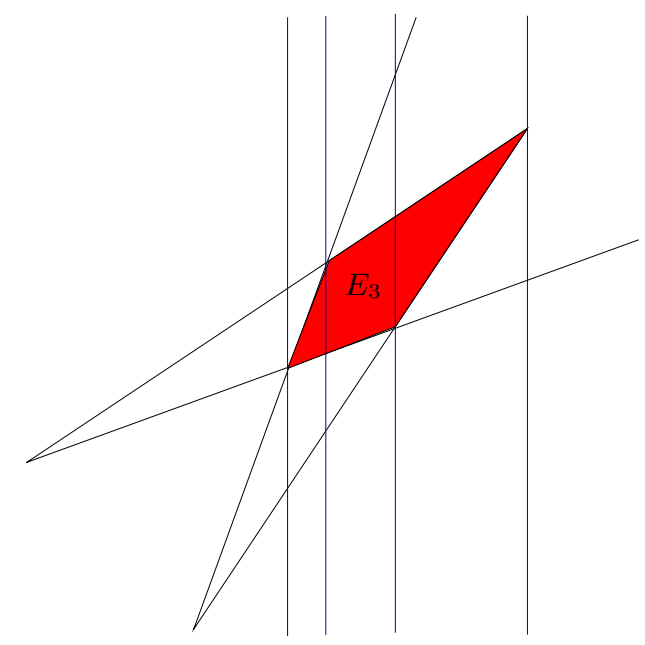

FIGURE 4.7: The $(a, b)$ yielding $H_{(a, b, 3)}$ with 5 isolated roots in the positive quadrant lie in the shaded region $E_{3}$. The vertices - intersected by the four vertical lines - are a subset of the critical points of the projection mapping $\bar{\nabla}_{\mathcal{H}(3)}$ onto the a-axis.

The boundary of $E_{3}$ thus appears to be a subset of the union of 4 convex arcs. More rigorously, it suffices to show that each of the 7 smooth sub-arcs above, obtained from the parametric formula of Example 2.16, is convex away from its cusps and nodes. This follows easily from computing the partial derivatives of the logarithms of our parametric formula and checking signs. So $E_{3}$ is indeed non-empty, star-convex, and in fact has 4 vertices determined by the polynomials mentioned in the statement of Theorem 1.2.

To conclude, we need only verify that $(44 / 31,44 / 31) \in E_{3}$. Instead of doing this via symbolic algebra, let us instead employ Smale's Alpha Theory, as summarized earlier: Clearly, we need only check partial derivatives of the $h_{i}$ up to order 6 , and a quick computation reveals that any $z=\left(z_{1}, z_{2}\right)$ chosen from one of the five following points satisfies $\alpha\left(H_{(44 / 31,44 / 31,3)}, z\right)<0.03$ :

$$
(0.584513273807,0.818672114695),(0.721441819886,0.757201442567) \text {, }
$$

$$
(0.740238978217,0.740238978217), \quad(0.757201442567,0.721441819886), \quad(0.818672114695,0.584513273807) .
$$

\footnotetext{
${ }^{7}$ Truthfully, we drew the mixed subdivisions of the pair $\left(\operatorname{Supp}\left(h_{1}\right), \operatorname{Supp}\left(h_{2}\right)\right)$, where $H_{(a, b, 3)}=\left(h_{1}, h_{2}\right)$. The latter diagrams can be interpreted as projections of the triangulations of $\mathcal{H}(3)$.
} 
Thus, each of these points is an approximate root of $H_{(44 / 31,44 / 31,3)}$. Also, since the roots of $H_{(44 / 31,44 / 31,3)}$ are clearly symmetric about the line $\{x=y\}$, we need only compute the $\alpha$-invariant 3 times. So, thanks to Theorem 4.4 (and Proposition 5.1 of the Appendix), we are done.

Remark 4.8. Alternatively, we could have simply used any Gröbner basis solver to get a rational univariate reduction for $H_{(44 / 31,44 / 31,3)}$. One could then use Sturm-Habicht sequences [Stu35, Hab48, Roy96, LM01] to find (certifiably correct) isolating intervals for the real roots. However, this naive Gröbnerian approach becomes infeasible for higher degree examples (cf. Note Added in Proof). $\diamond$

\section{The Proof of Theorem 1.2}

Here, we need only continue the development of the proof of Theorem 1.1 one step further: Since we already observed and proved the structure of the boundary of $E_{3}$ in Section 4, we need only verify that $E_{1}$ and $E_{2}$ are empty, and make two estimates concerning the size of $E_{3}$. The emptiness of $E_{2}$ follows from essentially the same techniques as we used for $E_{3}$, but the resulting computations (which we omit) are much simpler. The emptiness of $E_{1}$ follows directly from Bézout's Theorem: $H_{(a, b, 1)}$ - being a pair of bivariate quadratic polynomials - has no more than 4 non-degenerate isolated complex roots. Also, since we already know from Proposition 1.4 that $E_{3}$ is open, and our proof of Theorem 1.1 already showed $E_{3}$ to be non-empty, we clearly have $\operatorname{Area}\left(E_{3}\right)>0$.

To conclude, since the boundary curves of $E_{3}$ are concave, the area of $E_{3}$ is clearly bounded from above by the area of the convex hull of its vertices. Maple easily yields the estimate stated in our theorem. As for the estimate on the probability, we need only observe that the probability is in turn bounded above by the aforementioned area, times the value of the probability density function at the lower left vertex of $E_{3}$. (The lower left vertex is clearly the point of $E_{3}$ maximizing any function of the form $\alpha e^{\frac{-\left(x^{2}+y^{2}\right)}{\beta}}$, when $\alpha, \beta>0$.) Via Maple once again, we are done.

Note that our proof here in essence focussed on one reduced $\mathcal{A}$-discriminant chamber, for $\mathcal{A}=$ $\left[\begin{array}{cccccc}0 & -6 & -6 & -6 & -3 & -5 \\ 0 & 3 & 1 & 6 & 0 & 0 \\ 0 & 0 & 0 & 1 & 1 & 1\end{array}\right]$, while the proof in the last section described a collection of sets refining the true reduced $\mathcal{A}$-discriminant chambers. With the calculations we have already done, we can in fact describe just the relevant $\mathcal{A}$-discriminant chambers, and give the number of real roots for all non-degenerate $H_{(a, b, 3)}$. In particular, the intersection of $\mathbb{R}^{2} \cap \bar{\nabla}_{\mathcal{A}}$ with any sufficiently large square is, up to diffeotopy, the curve (possessing 3 isolated points) drawn below:

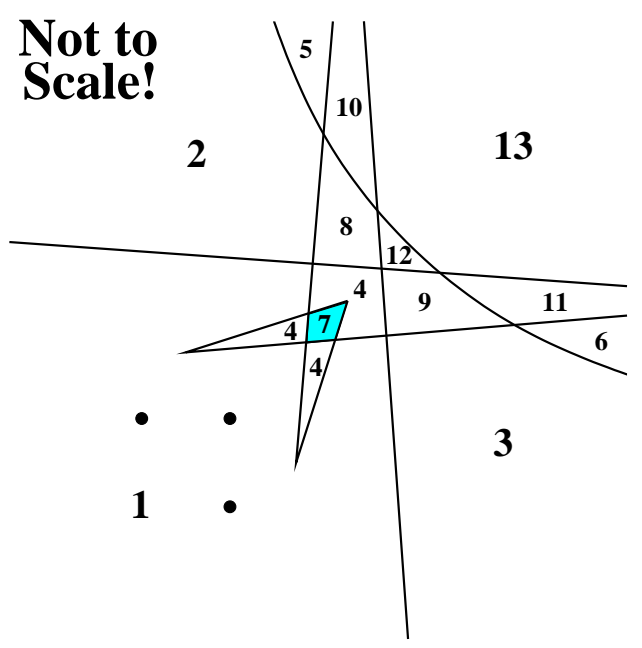

\begin{tabular}{|c|c|c|c|c|c|}
\hline region \# & ++ & -+ & -- & +- & rep. $(a, b)$ \\
\hline 1 & 1 & 0 & 0 & 0 & $(-1,-1)$ \\
\hline 2 & 1 & 0 & 0 & 2 & $(-1,5)$ \\
\hline 3 & 1 & 2 & 0 & 0 & $(5,-1)$ \\
\hline 4 & 3 & 0 & 0 & 0 & $\left(\frac{71}{50}, \frac{71}{50}\right)$ \\
\hline 5 & 1 & 0 & 2 & 2 & $(1,9)$ \\
\hline 6 & 1 & 2 & 2 & 0 & $(9,1)$ \\
\hline 7 & 5 & $\mathbf{0}$ & 0 & $\mathbf{0}$ & $\left(\frac{44}{31}, \frac{44}{31}\right)$ \\
\hline 8 & 3 & 0 & 0 & 2 & $\left(\frac{36}{25}, \frac{37}{25}\right)$ \\
\hline 9 & 3 & 2 & 0 & 0 & $\left(\frac{37}{25}, \frac{36}{25}\right)$ \\
\hline 10 & 3 & 0 & 2 & 2 & $\left(\frac{8}{5}, \frac{5}{2}\right)$ \\
\hline 11 & 3 & 2 & 2 & 0 & $\left(\frac{5}{2}, \frac{8}{5}\right)$ \\
\hline 12 & 3 & 2 & 0 & 2 & $\left(\frac{7}{4}, \frac{7}{4}\right)$ \\
\hline 13 & 3 & 2 & 2 & 2 & $(2,2)$ \\
\hline
\end{tabular}


Moreover, we see that the number of relevant $\mathcal{A}$-discriminant chambers is just 15 and, as detailed in the table above, there are just 13 possibilities for the quadruple consisting of the number of roots $H_{(a, b, 3)}$ in each real quadrant. A representative set of $(a, b)$ also appears in the table above.

\section{Appendix: Additional Computational Details}

- The polynomial $A(a)$, mentioned in Theorem 1.1 earlier, is the following univariate degree 36 polynomial with large integer coefficients:

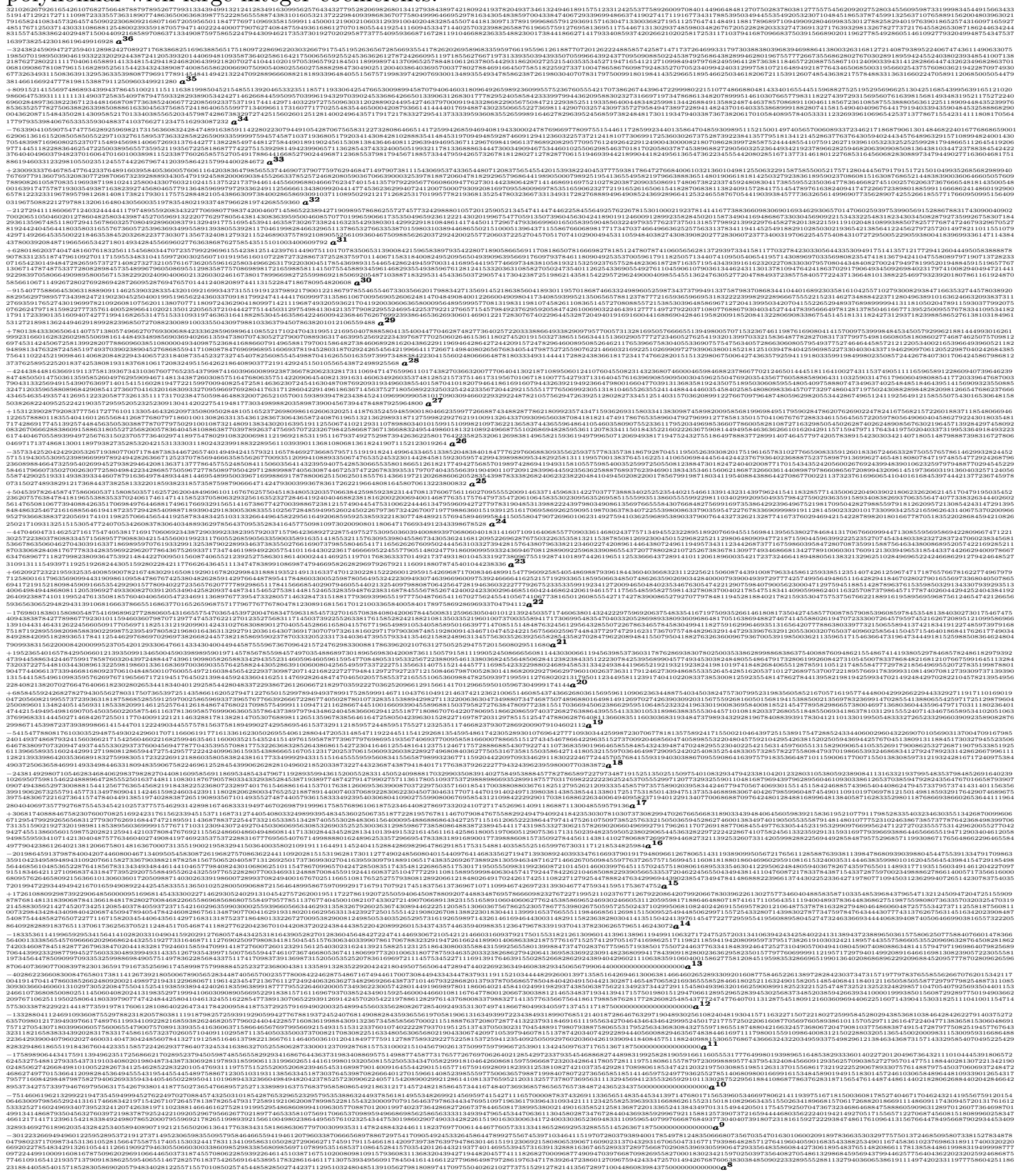




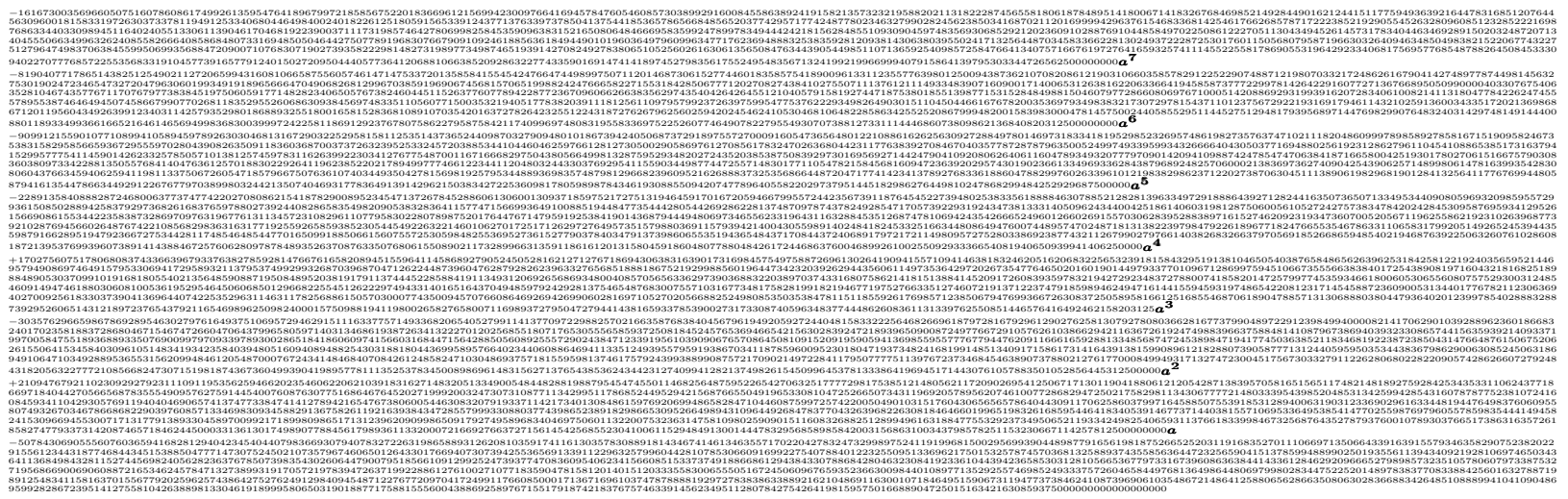

We point out that there are 254 digits per line, the largest coefficient of $A$ has 1460 digits, and $A$ is irreducible in $\mathbb{Z}[a]$ (verified via Maple).

- A bit later, in the proof of Theorem 1.1, we used the critical points method to study the complement of a reduced discriminant variety in $\left(\mathbb{R}^{*}\right)^{2}$. The locations (to 10 decimal places), and dispositions, of the underlying vertical lines $L_{i}$ and critical values are tabulated below:

\begin{tabular}{c|l}
-1.8562718399 & isolated point (on line $x=y$ ), root of $C^{*}$ \\
\hline-1.1581041767 & isolated point $\left(y\right.$ coordinate equal to $x$ coordinate of next isolated point), root of $A^{*}$ \\
\hline 0 & the $b$-axis \\
\hline 1.24487176148 & $a$-axis intersection \\
\hline 1.41544129863 & cusp (appearing in Figure 4.7), root of $B^{*}$ \\
\hline 1.41666026637 & cusp (appearing in Figure 4.7), root of $B^{*}$ \\
\hline 1.41767594900 & node (appearing in Figure 4.7), root of $C^{*}$ \\
\hline 1.41790510558 & node (appearing in Figure 4.7), root of $A^{*}$ \\
\hline 1.41821476967 & node (appearing in Figure 4.7), root of $A^{*}$ \\
\hline 1.41951679775 & cusp (appearing in Figure 4.7), root of $D^{*}$ \\
\hline 1.43683087662 & node, root of $A^{*}$ \\
\hline 1.47813022442 & node, root of $A^{*}$ \\
\hline 1.59316011321 & node, root of $C^{*}$ \\
\hline 1.60149022139 & node, root of $A^{*}$ \\
\hline 1.92733319557 & isolated point, $\left(y\right.$ coordinate equal to $x$ coordinate of preceding isolated point), root of $A^{*}$ \\
\hline 2.45494131563 & node, root of $A^{*}$ \\
\hline 2.47089273858 & node, root of $A^{*}$
\end{tabular}

- Finding the the aforementioned critical values took just over 30 minutes, using univariate resultants within Maple 10, on Rojas' dual 2.2Ghz Opteron Linux workstation with 4Gb memory. In particular, the locations were certified via use of the realroot command of Maple, which uses Sturm-Habicht Sequences [Stu35, Hab48, Roy96, LM01] to find intervals (with arbitrarily small size and rational endpoints) containing exactly the real algebraic numbers needed.

- Finding the location of the critical points, and thus certifying the isolated points, took close to 5 days. For the latter computation, we used the gsolve command, and took advantage of the fact that the underlying reduced discriminant is a composition of a low degree polynomial with low degree monomials (cf. Remark 4.5).

- The aforementioned refinement, $T^{*}$, of the reduced $\mathcal{A}$-discriminant complement turns out to have exactly 125 connected components. This was derived by picking rational numbers $a_{i}$ interlacing the 
locations of the vertical lines $L_{i}$, and then computing isolating intervals for the real roots of $R\left(a_{i}, b\right)$. Finding representative points within each such component then simply amounted to picking rational numbers $b_{j}^{(i)}$ interlacing these roots. In other words, the representative points are of the form $\left(a_{i}, b_{j}^{(i)}\right)$, and each such point was fed into a gsolve computation to find a rational univariate reduction (RUR) for $H_{\left(a_{i}, b_{j}^{(i)}, 3\right)}$. Each such RUR was then fed into Maple's realroot command, and in this way we found the number of real roots of every $H_{(a, b, 3)}$ in the reduced discriminant complement. One can definitely see in hindsight — after the proof of Theorem 1.2 in Section 5 that there was redundancy in our particular family of chosen $H_{(a, b, 3)}$.

- The approximations to the 5 isolated roots of $H_{(44 / 31,44 / 31,3)}$ in the positive quadrant were computed by Jan Verschelde (via his software package PHC-pack [Ver06]) during the October 2005 Midwest Algebraic Geometry Conference at Notre Dame University. The computation took a fraction of a second on a standard lap-top computer.

- For any square matrix $M$, let $\sigma(M)$ denote its largest singular value, i.e., the positive square root of the largest eigenvalue of $M^{T} M$. The computation of the $\alpha$-invariant for the Haas family can be greatly simplified by the following observation, which follows from a routine calculation by hand:

Proposition 5.1. Let $z=\left(z_{1}, z_{2}\right) \in \mathbb{C}^{2}, M_{-1}(z):=\left[\begin{array}{cc}6 z_{1}^{5} & \frac{132}{31} z_{2}^{2}-1 \\ \frac{132}{31} z_{1}^{2}-1 & 6 z_{2}^{5}\end{array}\right]^{-1}$, and for any $k \in\{2, \ldots, 6\}^{8}$

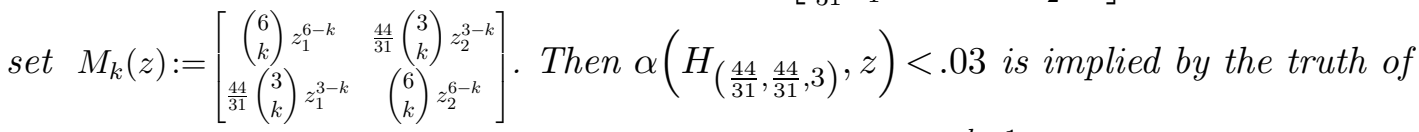

$$
\sigma\left(M_{-1}(z) M_{k}(z)\right)<\left(\frac{.03}{\left|M_{-1}(z) H_{(44 / 31,44 / 31,3)}(z)\right|}\right)^{k-1} \text { for all } k \in\{2, \ldots, 6\} .
$$

Note that the right-hand norm is a vector norm. The above simplification allows one to apply Theorem 4.4 using just rational operations, after some minor observations involving characteristic polynomials of $2 \times 2$ matrices. Using Maple's arbitrary precision arithmetic, one can then easily check that just 6 digits of accuracy (for the 5 putative approximate roots preceding Remark 4.8) suffices to yield $\alpha$ values below the critical threshold of 0.03 .

- A skeptical reader may certainly doubt the correctness of the underlying implementations of Gröbner bases, Sturm-Habicht sequences, and arbitrary precision arithmetic within Maple - but hopefully not all at once. This was one motivation behind our use of Alpha Theory.

- The locations of the vertical lines $L_{1}, \ldots, L_{18}$ - which were central in our proof of Theorems 1.1 and 1.2 - were independently verified by Bernard Mourrain (via the INRIA Sophia-Antipolis software package synaps) and Fabrice Roullier (via the INRIA Rocquencourt software package salsa), shortly after the September 2006 IMA Tutorial on Algebraic Geometric Methods in Engineering.

\section{Acknowledgements}

We would like to thank Thierry Zell for his quick and detailed answers to questions on Pfaffian functions; Frederic Bihan and Frank Sottile for informing the authors of [BS06]; Bernard Mourrain, Fabrice Roullier, and Jan Verschelde for additional independent verification of some of our underlying calculations; Michel Coste for pointing out important earlier work on the finiteness of topological types of fewnomial functions and zero sets [Cos98]; and the anonymous referee for

\footnotetext{
${ }^{8}$ When $i \notin\{0, \ldots, j\}$, we define $\left(\begin{array}{l}j \\ i\end{array}\right)=0$.
} 
valuable suggestions that clarified and improved this paper. We would also like to thank IMA (the Institute for Mathematics and its Applications, at the University of Minnesotta, Minneapolis), for their hospitality during the completion of this work. The second author is also grateful for the support of M. Danny Rintoul III during the early stages of this work, while he was visiting the Computational Biology Group at Sandia National Laboratories.

Finally, we would like to wish Professor Khovanskii a very happy $60 \underline{\text { th }}$ birthday. We have long admired his work and his tremendous talent for turning even the most difficult ideas into simple and beautiful explanations.

Note Added in Proof: In July 2006, Andrew Niles, an NSF sponsored undergraduate student (DMS-0552610) in the second author's 2006 REU class at Texas A $6 M$ University, found a $2 \times 2$ real polynomial system - consisting of a degree 6 trinomial and a degree 141 tetranomial — with exactly 7 isolated roots in $\mathbb{R}_{+}^{2}$.

Also, around the same time Martin Avendaño, a Ph.D. student at the University of Buenos Aires, found a new upper bound for the number of real intersections of a line and an $m$-nomial [Kri06]. By [LRW03, Sec. 3, Lemma 1], Avendaño's result immediately implies that $2 \times 2$ real fewnomial systems of type $(3, m)$ never have more than $6 m-1$ isolated roots in $\mathbb{R}_{+}^{2}$. The latter bound is a serious improvement over the best previous bound of $2^{m}-2$ [LRW03, Thm. 1 (a)]. $\diamond$

\section{References}

[BV06] Basu, Saugata and Vorobjov, Nicolai N., "On the Number of Homotopy Types of Fibres of a Definable Map," Journal of the London Mathematical Society, to appear. Also available as Math ArXiV preprint math.AG/0605517.

[BRS06] Bihan, Frederic; Rojas, J. Maurice; and Stella, Casey, "First Steps in Algorithmic Fewnomial Theory," submitted for publication. Also available as Math ArXiV preprint math. AG/0411107.

[BS06] Bihan, Frederic and Sottile, Frank, "New Fewnomial Upper Bounds from Gale Dual Polynomial Systems," Moscow Mathematical Journal, to appear. Also available as Math ArXiV preprint math.AG/0609544 .

[BCSS98] Blum, Lenore; Cucker, Felipe; Shub, Mike; and Smale, Steve, Complexity and Real Computation, Springer-Verlag, 1998.

[CSMP03] Castro, David; San Martín, Jorge; and Pardo, Luis-Miguel, "Systems of rational polynomial equations have polynomial size approximate zeros on the average," J. Complexity 19 (2003), pp. 161-209.

[CG84] Chistov, Alexander L., and Grigoriev, Dima Yu, "Complexity of Quantifier Elimination in the Theory of Algebraically Closed Fields," Lect. Notes Comp. Sci. 176, Springer-Verlag (1984).

[CZ02] Cohen, Paula B. and Zannier, Umberto, "Fewnomials and intersections of lines with real analytic subgroups in $\mathbf{G}_{m}^{n}$," Bull. London Math. Soc. 34 (2002), no. 1, pp. 21-32.

[Cos98] Coste, Michel, "Topological types of fewnomials," Singularities Symposium — Łojasiewicz 70 (Kraków, 1996; Warsaw, 1996), pp. 81-92, Banach Center Publ., 44, Polish Acad. Sci., Warsaw, 1998. 
[CD06] Cueto, María Angélica and Dickenstein, Alicia, Some results on inhomogeneous discriminants, to appear: Proc. XVI CLA, Biblioteca de la Revista Matemática Iberoamericana.

[DFS05] Dickenstein, Alicia; Feichtner, Eva Maria; and Sturmfels, Bernd, "Tropical Discriminants," Math ArXiV preprint math.AG/0510126 .

[DS02] Dickenstein, Alicia and Sturmfels, Bernd, "Elimination Theory in Codimension Two," Journal of Symbolic Computation (2002) 34, pp. 119-135.

[vdD98] van den Dries, Lou, Tame topology and o-minimal structures, London Mathematical Society Lecture Note Series, 248, Cambridge University Press, Cambridge, 1998.

[Ful93] Fulton, William, Introduction to Toric Varieties, Annals of Mathematics Studies, no. 131, Princeton University Press, Princeton, New Jersey, 1993.

[GV01] Gabrielov, Andrei and Vorobjov, Nicolai, "Complexity of cylindrical decompositions of subPfaffian sets," Effective methods in algebraic geometry (Bath, 2000), J. Pure Appl. Algebra 164 (2001), no. 1-2, pp. 179-197.

[GVZ04] Gabrielov, Andrei; Vorobjov, Nikolai; and Zell, Thierry, "Betti Numbers of Semialgebraic and Sub-Pfaffian Sets," J. London Math. Soc. 69 (2004), pp. 27-43.

[GKZ94] Gel'fand, Israel Moseyevitch; Kapranov, Misha M.; and Zelevinsky, Andrei V.; Discriminants, Resultants and Multidimensional Determinants, Birkhäuser, Boston, 1994.

[GK03] Algebraic Geometry and Geometric Modelling, Proceedings of a conference in Vilnius, Lithuania (July 29-August 2, 2002), Contemporary Mathematics, (edited by Ron Goldman and Rimvydas Krasauskas), vol. 334, American Mathematical Society, 2003.

[Haa02] Haas, Bertrand, “A Simple Counter-Example to Kushnirenko's Conjecture," Beiträge zur Algebra und Geometrie, Vol. 43, No. 1, pp. 1-8 (2002).

[Hab48] Habicht, Walter, "Eine Verallgemeinerung des Sturmschen Wurzelzhlverfahrens," Comment. Math. Helv. 21 (1948), pp. 99-116.

[Har76] Harnack, Carl Gustav Axel, "Über die Vielfaltigkeit der ebenen algebraischen Kurven," Math. Ann. 10 (1876), pp. 189-199.

[Kal03] Kaloshin, V., "The existential Hilbert 16-th problem and an estimate for cyclicity of elementary polycycles," Invent. Math. 151 (2003), no. 3, pp. 451-512.

[Kap91] Kapranov, Misha, "A characterization of A-discriminantal hypersurfaces in terms of the logarithmic Gauss map," Mathematische Annalen, 290, 1991, pp. 277-285.

[Kho80] Khovanskii, Askold G., "On a Class of Systems of Transcendental Equations," Dokl. Akad. Nauk SSSR 255 (1980), no. 4, pp. 804-807; English transl. in Soviet Math. Dokl. 22 (1980), no. 3 .

[Kho91] _ Fewnomials, AMS Press, Providence, Rhode Island, 1991.

[Koi97] Koiran, Pascal, "Randomized and Deterministic Algorithms for the Dimension of Algebraic Varieties," Proceedings of the $38^{\text {th }}$ Annual IEEE Computer Society Conference on Foundations of Computer Science (FOCS), Oct. 20-22, 1997, ACM Press. 
[Kri06] Krick, Teresa, personal communication, at the Institute for Mathematics and its Applications (University of Minnesotta, Minneapolis), Sept. 15, 2006.

[LRW03] Li, Tien-Yien; Rojas, J. Maurice; and Wang, Xiaoshen, "Counting Real Connected Components of Trinomial Curves Intersections and m-nomial Hypersurfaces," Discrete and Computational Geometry, 30:379-414 (2003).

[LM01] Lickteig, Thomas and Roy, Marie-Francoise, "Sylvester-Habicht Sequences and Fast Cauchy Index Computation," J. Symbolic Computation (2001) 31, pp. 315-341.

[Loe91] Loeser, François, "Polytopes secondaires et discriminants," Séminaire Bourbaki, Vol. 1990/91. Astérisque No. 201-203 (1991), Exp. No. 742, pp. 387-420 (1992).

[OK00] Orevkov, S. Yu. and Kharlamov, V. M., "Asymptotic growth of the number of classes of real plane algebraic curves when the degree increases," J. of Math. Sciences, 113 (2003), no. 5, pp. 666-674.

[PT05] Passare, Mikael and Tsikh, August, "Amoebas: their spines and their contours," Idempotent mathematics and mathematical physics, Contemp. Math., v. 377, Amer. Math. Soc., Providence, RI, 2005, pp. 275-288.

[PT04] Passare, Mikael and Tsikh, August, "Algebraic equations and hypergeometric series," in: The Legacy of Niels Henrik Abel, Springer-Verlag, Berlin; Heidelberg, 2004.

[Per05] Perrucci, Daniel, "Some Bounds for the Number of Components of Real Zero Sets of Sparse Polynomials," Discrete and Computational Geometry, to appear.

[Pla84] Plaisted, David A., "New NP-Hard and NP-Complete Polynomial and Integer Divisibility Problems," Theoret. Comput. Sci. 31 (1984), no. 1-2, 125-138.

[Roj03] Rojas, J. Maurice, "Why Polyhedra Matter in Non-Linear Equation Solving," paper corresponding to an invited talk delivered at a conference on Algebraic Geometry and Geometric Modelling (Vilnius, Lithuania, July 29-August 2, 2002), Contemporary Mathematics, vol. 334, pp. 293-320, AMS Press, 2003.

[Roj04] Rojas, J. Maurice, "Arithmetic Multivariate Descartes' Rule," American Journal of Mathematics, vol. 126, no. 1, February 2004, pp. 1-30.

[RY05] Rojas, J. Maurice and Ye, Yinyu, "On Solving Sparse Polynomials in Logarithmic Time," Journal of Complexity, special issue for the 2002 Foundations of Computation Mathematics (FOCM) meeting, February 2005, pp. 87-110.

[RS98] Rosenthal, Joachim and Sottile, Frank, "Some remarks on real and complex output feedback," Systems Control Lett. 33 (1998), no. 2, pp. 73-80.

[Roy96] Roy, Marie-Françoise, "Basic Algorithms in Real Algebraic Geometry and their Complexity: from Sturm's Theorem to the Existential Theory of Reals," Lectures in Real Geometry (Madrid, 1994), pp. 1-67, de Gruyter Exp. Math., 23, de Gruyter, Berlin, 1996.

[Sma86] Smale, Steve, "Newton's Method Estimates from Data at One Point," The Merging of Disciplines: New Directions in Pure, Applied, and Computational Mathematics (Laramie, Wyo., 1985), pp. 185-196, Springer, New York, 1986. 
[Sma00] "Mathematical Problems for the Next Century," Mathematics: Frontiers and Perspectives, pp. 271-294, Amer. Math. Soc., Providence, RI, 2000.

[SL54] Smith, David Eugene and Latham, Marcia L., The Geometry of René Descartes, translated from the French and Latin (with a facsimile of Descartes' 1637 French edition), Dover Publications Inc., New York (1954).

[Sto98] Storjohann, Arne, "Computing Hermite and Smith normal forms of triangular integer matrices," Linear Algebra Appl. 282 (1998), no. 1-3, pp. 25-45.

[Stu35] Sturm, C., "Mémoire sur la résolution des équations numériques," Inst. France Sc. Math. Phys., 6 (1835).

[VG03] Vakulenko, Sergey and Grigoriev, Dmitry, "Complexity of gene circuits, Pfaffian functions and the morphogenesis problem," C. R. Math. Acad. Sci. Paris 337 (2003), no. 11, pp. 721-724.

[Ver06] Verschelde, Jan, PHC-Pack, http://www.math.uic.edu/ jan/download.html .

[Wil99] Wilkie, A. J., "A theorem of the complement and some new o-minimal structures," Selecta Math. (N.S.) 5 (1999), no. 4, pp. 397-421. 